Research Article

\title{
The Influence of Three Statistical Variables on Self-Similarity in Complex Networks
}

\author{
Mingli Lei, Lirong Liu $(\mathbb{D}$, and Daijun Wei 1 \\ School of Science, Hubei University for Nationalities, Enshi 445000, China \\ Correspondence should be addressed to Daijun Wei; prof.wei@hotmail.com
}

Received 9 September 2019; Revised 28 October 2019; Accepted 30 October 2019; Published 13 January 2020

Academic Editor: Filippo Cacace

Copyright (c) 2020 Mingli Lei et al. This is an open access article distributed under the Creative Commons Attribution License, which permits unrestricted use, distribution, and reproduction in any medium, provided the original work is properly cited.

\begin{abstract}
The reason for the self-similarity property of complex network is still an open issue. In this paper, we focus on the influence of degree, betweenness, and coreness on self-similarity of complex network. Some nodes are removed from the original network based on the definitions of degree, betweenness, and coreness in the ascending and descending order. And then, some new networks are obtained after removing nodes. The self-similarities of original network and new networks are compared. Moreover, two real networks are used for numerical simulation, including a USAir network and the yeast protein interaction (YPI) network. The effects of the three statistical variables on the two real networks are considered. The results reveal that the nodes with large degree and betweenness have great effects on self-similarity, and the influence of coreness on self-similarity is small.
\end{abstract}

\section{Introduction}

Self-similarity property of complex systems has attracted the attention of many researchers and has been widely studied [1-5]. In 1997, the self-similarity property of World Wide Web (WWW) network transfer was identified [6]. In 2005, the self-similarity property of complex network has been found while the network was renormalized by using the box covering method [7]. In [8], the box covering algorithm was designed to measure the fractal of real networks, which is named as box dimension. Box covering and box dimension of complex networks have been widely studied [9-12]. On the one hand, box dimension and box covering algorithms have been extended or modified. For example, the box covering algorithm was modified to calculate the box dimension of the weighted complex networks by Wei et al. [9]. In [13], not only box dimension was modified by considering the numbers of nodes in the box but also the fractal dimension of weighted fractal networks was modified in that the number of iterations and weighting factors are considered. A weighted box covering algorithm has been proposed by Song et al., and it has been used to study the fractal property of weighted network and multifractal property [10]. On the other hand, many other fractal dimensions have also been proposed to quantitatively measure the self-similarity of complex networks $[13,14]$, such as volume dimension and information dimension [11, 12, 15-17].

For complex networks, only a small portion of network has self-similarity. The self-similarity does not exist in all complex networks [18]. Although many fractal dimensions are applied to measure the self-similarity, when the selfsimilarity networks are measured by these fractal dimensions, all methods have the same characteristics, i.e., the fractal dimension $d_{\mathrm{B}}$ satisfies power law distribution $N_{\mathrm{B}} \approx \ell_{\mathrm{B}}^{-d_{\mathrm{B}}}$, where $l_{\mathrm{B}}$ and $N_{\mathrm{B}}$ are the size of box covering network and the number of boxes needing to cover network within the box size $l_{\mathrm{B}}$, respectively [7]. There are many selfsimilarities, including structure self-similarity [7], volume self-similarity [15], and information self-similarity [11,12]. The self-similarity between the whole and part of network is represented by the structure self-similarity, which is usually measured by the box dimension Dim. The relationship between the size of the box covering network and the number of boxes covering the network within the box size $l_{\mathrm{B}}$ is applied to define box dimension Dim [7,8]. The volume self-similarity is measured by the volume dimension $d_{\mathrm{d}}$. The distance of covering network and the sum of the degree of nodes in the box within the distance of $l_{\mathrm{B}}$ are applied to 
measure the volume self-similarity [15]. Information selfsimilarity indicates the similarity between the information and local information in the network and is measured by the information dimension $d_{\mathrm{I}}$, which denotes the relationship between the size of box covering network and the amount of information contained in the box of size $l_{\mathrm{B}}[11,12]$.

However, the fundamental problem is the structure evolution for the self-similarity of complex network. Therefore, many researchers paid attention to the formation mechanism of complex networks [7, 18-20]. In [18], the fractal and nonfractal networks were studied by the renormalization mechanism and the results showed that the key reason for the formation of fractal structure is the strong repulsion among the large-degree nodes. The reason for selfsimilarity is heterogeneity of connected nodes in complex networks. In this view, heterogeneity is due to the reason that the nodes of large degree are connected with nodes of small degree, which is accepted by lots of researchers $[21,22]$. Moreover, the influence of large-degree nodes on network has been researched [23, 24].

There are many other indicators to reveal the importance of nodes in complex networks [25-28], including betweenness and coreness $[29,30]$. For the yeast protein interaction (YPI) network, the degree of some nodes is low, while betweenness is high. Thus, nodes with high betweenness play a key role in network [29]. Moreover, on the basis of betweenness centrality, random links are added to study the relationship between fractal and nonfractal properties of real scale-free networks. Kitsak et al. found that the correlation between degree and betweenness centrality of fractal networks is much weaker than of nonfractal networks [31]. The betweenness centrality was extended by Gao et al.; they combined the physarum centrality and $\mathrm{k}$ - core index to identify influential nodes in weighted network [32]. In addition, coreness $(\mathrm{k}$-core) was introduced to measure the influence of nodes. Kim et al. studied the fractal property and self-similarity of scale-free networks under the consideration of the $\mathrm{k}$-core and found that because of the increase of $\mathrm{k}$-core, fractal dimensions of networks were changed [33].

The topological structure of complex networks and the influence of nodes removal on the network were considered [23]. Residual closeness centrality was proposed by Dangalchev and applied to study the effects of nodes removal on network [34]. In order to identify the node importance, the Jaccard similarity coefficient is introduced to measure the neighborhood similarity. The robustness of the network is determined by removing nodes [28]. Restrepo et al. had studied the changes of maximum eigenvalue and the maximum eigenvalue was also applied to measure the dynamical influence of nodes and edges after nodes removal of complex networks [35]. Removing nodes from the original network is a dynamical behavior for studying the property of complex networks. On the one hand, studying the property of subnetwork is important when whole nodes and edges of the original network have been known by comparing the property of subnetwork with the original network [7, 31]. On the other hand, the topological property of whole network is uncertain in the real network. In order to infer the topological property of whole network, the property of subnetwork is usually studied. For example, Lü et al. studied the link prediction of complex networks by considering the links among existing nodes to predict links between unknown nodes [36]. In [37], a network growth model and a sparse network with determined diameter are obtained, and the average node degree of the network increases logarithmically with the growth of the network size. In disease areas in biology, the protein-protein interaction networks were studied, and the known interaction of among proteins was discussed and used to predict the interaction in other unknown proteins and to cure disease [38]. Many of the above methods study the changes of network structure from the perspective of adding edges.

However, the influence of nodes removal on self-similarity of the network is rarely studied. In this paper, the influence of three statistical variables on self-similarity is discussed by removing nodes gradually. The effects of degree, betweenness, and coreness on self-similarity in complex networks are, respectively, discussed, after removing nodes from the original network. With nodes removal, the self-similarities of the original network and subnetwork are compared. The degree of nodes is ranked in the descending and ascending order. Then, first $5 \%$ of nodes are removed for every rank and the change of box dimension Dim of the subnetwork is discussed. At the same time, the cluster coefficient is used to measure the changes of network structure. Moreover, betweenness and coreness of nodes are discussed in the same way. In addition, two real networks (i.e., a USAir network $[9,39]$ and the YPI network $[40,41])$ are used for numerical simulation.

The paper is organized as follows: basic concepts are introduced in Section 2. In Section 3, the influence of degree, betweenness, and coreness on self-similarity of network is discussed with nodes removal. In Section 4, two real networks are used for numerical simulation. Summarization is made in Section 5.

\section{Preliminaries}

In this section, the definitions of three statistical variables (i.e., degree, betweenness, and coreness) and the self-similarity property of complex networks are introduced. For the self-similarity property of complex networks, the box covering algorithm and fractal dimension are described.

\subsection{Three Statistical Variables of Nodes}

2.1.1. Degree. The degree of node $i$ is denoted by $k_{i}$, that is, the number of edges linked to the node $i$. The degree of node is a fundamental measure to describe complex networks. The definition of degree of node $i$ for the undirected network is given as follows [42]:

$$
k_{i}=a_{i i}^{(2)}=\operatorname{tr}\left(A^{2}\right)
$$

where $a_{i i}^{(2)}$ is the diagonal element of the second power of adjacency matrix $A^{2}$ and $\operatorname{tr}\left(A^{2}\right)$ is the trace of matrix $A^{2}$. In fact, the degree $k_{i}$ of node $i$ is also calculated by another way, that is, 


$$
\sum_{j=1}^{N} a_{i j}=\sum_{j=1}^{N} a_{j i}
$$

where $N$ is the total number of nodes.

From equations (1) and (2), $k_{i}$ reveals the relationship between the links of node $i$ and others.

2.1.2. Betweenness. Betweenness is another index to measure the importance of nodes. Although the degree of some nodes is small, these nodes act as intermediate bridges in the network. If these nodes are removed, the structure of network is destroyed. Betweenness of node $i$ is denoted by $B_{i}$ [42]:

$$
B_{i}=\sum_{\substack{1 \leq i \leq N \\ j \neq i \neq l}} \frac{n_{j l}(i)}{n_{j l}},
$$

where $n_{j l}$ is the number of the shortest paths between nodes $j$ and $l ; n_{j l}(i)$ is the number of the shortest paths between nodes $j$ and $l$ through node $i$; and $N$ is the total number of nodes.

The shortest paths between nodes $i$ and $j$ are not adjacent in the network. If there are many shortest paths through a node, betweenness of the node is large, the role of the "bridge" is more obvious, and the node is regarded as more important in the network [42].

2.1.3. Coreness. The $\mathrm{k}$-core of a network is the remaining subnetwork after the nodes with a degree less than $k$ are removed [43]. The number of nodes in the subnetwork is equal to the size of the coreness. If a node has $\mathrm{k}-$ core and is removed from $(k+1)$ - core, the coreness of this node is $k$. The maximum coreness of node is regarded as the coreness of network. If the degree of all nodes is 1 , the coreness of the network is 1 [42].

2.1.4. Cluster Coefficient. The neighbor nodes of network may be connected to each other, and the degree of neighbor nodes cluster was defined as the cluster coefficient [44]. The tightness of network was always quantified by the cluster coefficient. The larger the cluster coefficient, the closer the relationships among network nodes [45]. Cluster coefficient is defined as follows:

$$
C_{i}=\frac{E_{i}}{C_{k_{i}}^{2}}=\frac{2 E_{i}}{k_{i}\left(k_{i}-1\right)},
$$

where $k_{i}$ and $E_{i}$ are the degree and the number of edges between adjacent nodes of node $i$, respectively.

The average cluster coefficient is always used to reflect the tightness of network. In this paper, the average cluster coefficient is used to measure the tightness of network, that is,

$$
\langle C\rangle=\frac{1}{N} \sum_{i=1}^{N} C_{i},
$$

where $N$ is the number of nodes in total.

\subsection{Method of Self-Similarity for Networks}

2.2.1. Measures of Network Self-Similarity. When the selfsimilarity of network is discovered [7], further research shows that there are many types of self-similarity of network, including structure self-similarity [7], volume self-similarity [15], and information self-similarity $[11,12][16,17]$. At the same time, many methods are proposed to measure the selfsimilarity, such as box dimension $[7,8]$, volume dimension [15], and information dimension [11, 12]. Although there are many self-similar metrics, many fractal dimensions are improved based on the following equation $[7,8]$ :

$$
d_{\text {fract }}=-\frac{\ln N_{\mathrm{B}}}{\ln l_{\mathrm{B}}} .
$$

For box dimension Dim, $l_{\mathrm{B}}$ is the size of box needing to cover the network and $N_{\mathrm{B}}$ is the number of boxes covering the network within the box of size $l_{\mathrm{B}}$ in equation (6). The box dimension Dim is usually applied to measure the structure self-similarity of the network, which will be introduced in the next section [8]. Based on equation (6), by considering the sum of degrees of nodes $V_{\mathrm{D}}(r)$ within a given distance range $r$, a volume dimension is defined to measure the volume self-similarity of network, which is denoted as follows [15]:

$$
\begin{aligned}
V_{\mathrm{D}}(r) & \approx r^{d_{\mathrm{d}}}, \\
d_{\mathrm{d}} & =-\frac{\ln V_{\mathrm{D}}}{\ln r},
\end{aligned}
$$

Besides degrees of nodes being considered to measure the self-similarity, the information $I \prime(l)$ contained in a box of size $l$ is considered to measure the information selfsimilarity of network. The information dimension $d_{I}^{\prime}$ is defined [11] as follows:

$$
d_{I}^{\prime}=-\lim _{l \longrightarrow 0} \frac{I^{\prime}(l)}{\ln (l)}=\lim _{l \longrightarrow 0} \frac{\sum_{i=1}^{N_{b}} p_{i}^{\prime}(l) \ln p_{i}^{\prime}(l)}{\ln (l)} .
$$

In this paper, the box dimension Dim is used to measure the structure self-similarity of networks. If the proportional relationship of equation (6) is not satisfied, the corresponding network is not the self-similarity network and the network does not have the self-similarity characteristic.

2.2.2. Strictly Iterative Fractal Network (SFnet). Strictly iterative fractal network is a standard self-similarity fractal network and has a good power law distribution. In this paper, a strictly iterative fractal network is constructed, and the network construction steps are as follows [46]:

(1) Selecting four nodes as the seeds, one is regarded as the center, the other is in the vicinity, and three edges are used to connect the three adjacent nodes to the center node. The original network is shown in Figure 1(a). 


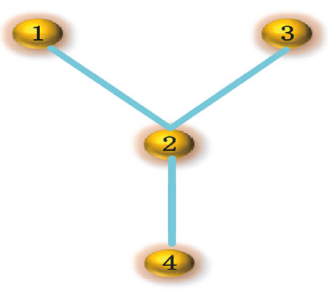

(a)

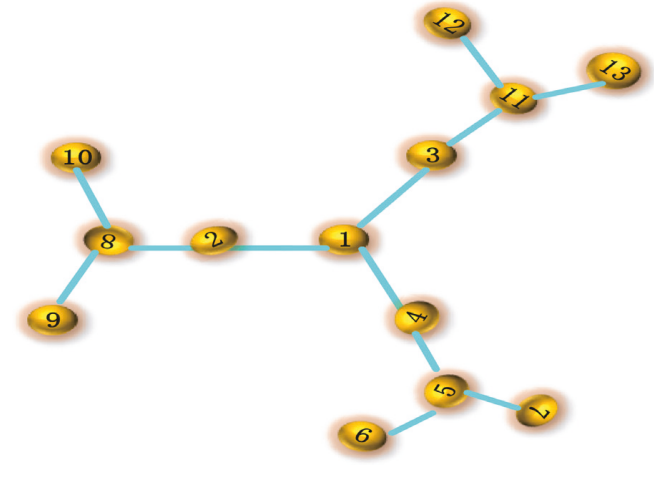

(b)

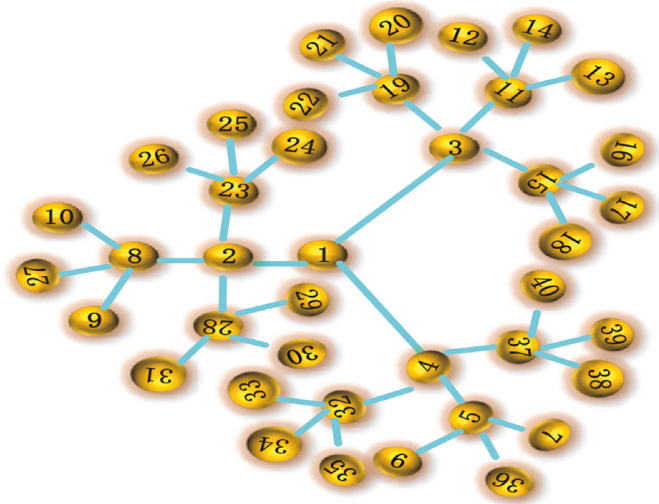

(c)

Figure 1: The process of constructing an SFnet.

(2) Each node of the original network is the center, and every center is connected with three nodes. A new network is shown in Figure 1(b).

(3) Repeating the second step $n-1$ times.

In this paper, $n$ is set as 4 . An SFnet is constructed which has 120 nodes and 121 edges as shown in Figure 2.

The self-similarity of the network is the similarity between the whole and part of network, and there is no difference in time or space. Fractal dimension is often used to measure network self-similarity. In this paper, the box dimension Dim is applied to measure the self-similarity of the network. The box dimension Dim will be introduced in the next section.

2.2.3. Box Covering Algorithm of Fractal Dimension. The box covering method has been widely used to explore the traditional fractal geometry. The fractal dimension began at the graph in the Euclidean space. Song et al. extended it to complex networks [7]. The size of the box covering network $l^{\prime}$ and the number of boxes required to cover the network $N_{\mathrm{B}}$ have the power law distribution, i.e., $N_{\mathrm{B}} \sim l^{-d}$. In this paper, the fractal dimension is the box dimension Dim, and it is calculated by using equation (9). Box dimension Dim is calculated by the box covering algorithm, and it is as follows [8]:

(1) Calculating the diameter of a network, i.e., the maximum of all the shortest paths of network.

(2) Covering the network with the box of size 1 and recording the number of boxes needed to cover the network.

(3) Each time, the box size is increased by 1 from the previous time to cover the network, and the corresponding number of boxes is recorded until the box size is the diameter of the network.

(4) Displaying the size of the box and the number of boxes required for the corresponding coverage on the double logarithmic graph. The slope is the network box dimension by least squares fitting.
The box dimension Dim is defined by the following equation:

$$
\operatorname{Dim}=-\frac{\ln N_{\mathrm{B}}\left(l_{\mathrm{B}}\right)}{\ln l_{\mathrm{B}}},
$$

where $l_{\mathrm{B}}$ is the box size and $N_{\mathrm{B}}\left(l_{\mathrm{B}}\right)$ is the number of boxes while a box of size $l_{\mathrm{B}}$ is used to cover the network.

\section{Effects of Three Statistical Variables on Self-Similarity}

In this section, the effects of degree, betweenness, and coreness on self-similarity are discussed with gradual nodes removal. Degree, betweenness, and coreness are, respectively, sorted in the descending and ascending order. Then, the first $5 \%$ of nodes are removed for every rank. Moreover, the change of box dimension of subnetworks is discussed. Some of symbols mentioned later are explained here and are listed in Table 1.

3.1. Influence of Degree on Self-Similarity. Hub node is defined as the node with the largest degree of a network. In Ref. [18], a probability $e$ is defined and used to measure the level of hub attraction. Networks with higher probability $e$ tend to be nonfractal. On the contrary, lower probability networks are close to be fractal [18]. In this section, the influence of degree on self-similarity for a constructed strictly fractal network (SFnet) is discussed. The constructed network has 121 nodes and 120 edges which is shown in Figure 3. Nodes are, respectively, sorted in the descending and ascending order based on the definition of degree. Then, the first $5 \%$ of nodes are removed and the steps of sorting and nodes removal are repeated, until $45 \%$ of nodes are removed in the descending order and $60 \%$ of nodes are removed in the ascending order. Moreover, box dimension and cluster coefficient of subnetworks are calculated.

The influence of nodes removal by means of degree descending order on the SFnet is studied. The box dimension $\operatorname{Dim}_{\mathrm{d}}$ and cluster coefficient $\langle C\rangle_{\mathrm{d}}$ of subnetworks are, respectively, calculated by using equations (5) and (9), which 


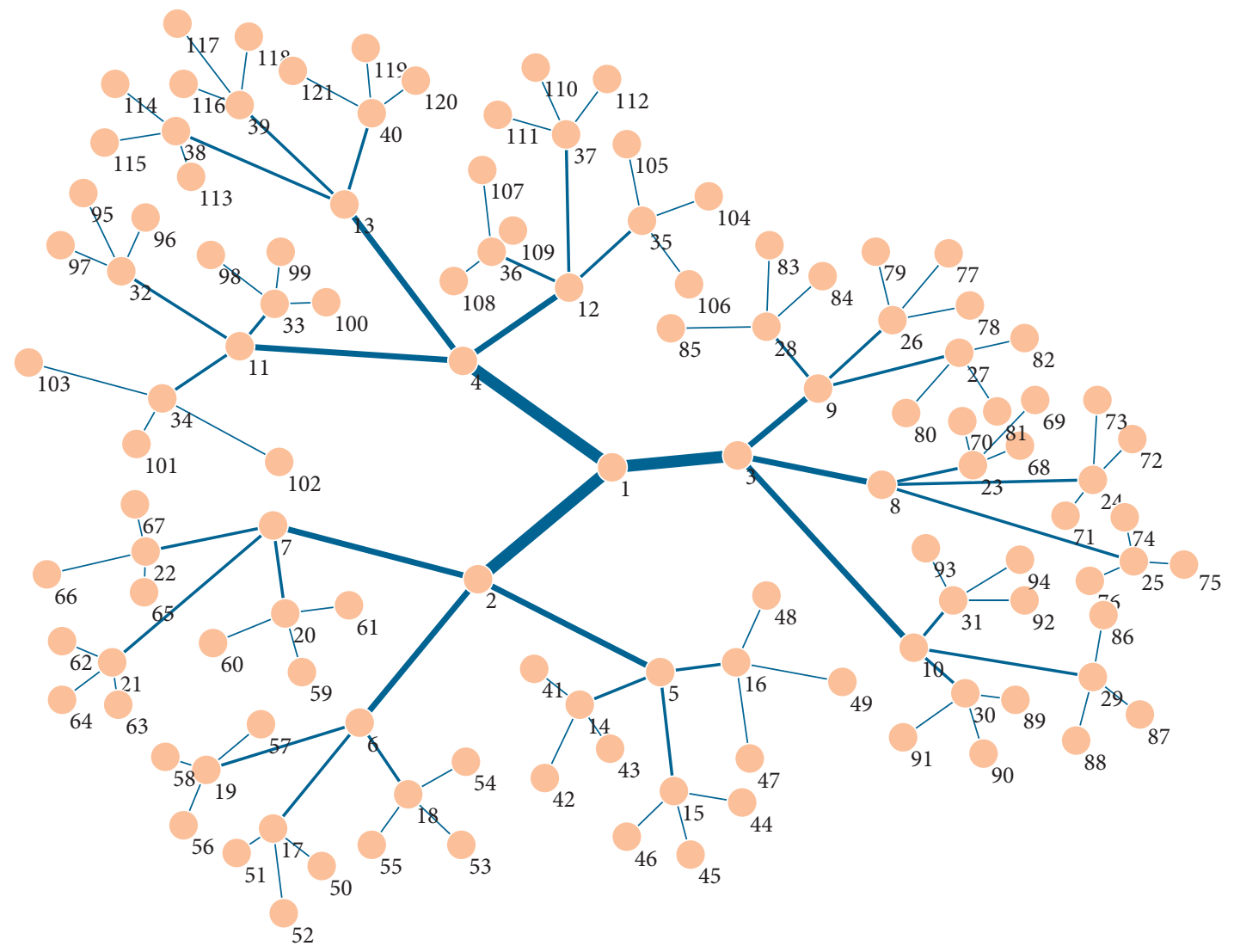

FIGURE 2: A constructed SFnet.

TABLE 1: Some symbolic explanations.

\begin{tabular}{|c|c|}
\hline Symbol & Explanations \\
\hline $\operatorname{Dim}_{d}$ & $\begin{array}{c}\text { Box dimension of network with nodes removal by } \\
\text { degree in the descending order }\end{array}$ \\
\hline $\operatorname{Dim}_{b}$ & $\begin{array}{l}\text { Box dimension of network with nodes removal by } \\
\text { betweenness in the descending order }\end{array}$ \\
\hline $\operatorname{Dim}_{c}$ & $\begin{array}{l}\text { Box dimension of network with nodes removal by } \\
\text { coreness in the descending order }\end{array}$ \\
\hline$\langle C\rangle_{\mathrm{d}}$ & $\begin{array}{c}\text { Cluster coefficient of network with nodes removal by } \\
\text { degree in the descending order }\end{array}$ \\
\hline$\langle C\rangle_{\mathrm{b}}$ & $\begin{array}{c}\text { Cluster coefficient of network with nodes removal by } \\
\text { betweenness in the descending order }\end{array}$ \\
\hline$\langle C\rangle_{\mathrm{c}}$ & $\begin{array}{c}\text { Cluster coefficient of network with nodes removal by } \\
\text { coreness in the descending order }\end{array}$ \\
\hline $\operatorname{Dim}_{d}^{\prime}$ & $\begin{array}{c}\text { Box dimension of network with nodes removal by } \\
\text { degree in the ascending order }\end{array}$ \\
\hline $\operatorname{Dim}_{b}^{\prime}$ & $\begin{array}{l}\text { Box dimension of network with nodes removal by } \\
\text { betweenness in the ascending order }\end{array}$ \\
\hline $\operatorname{Dim}_{c}^{\prime}$ & $\begin{array}{l}\text { Box dimension of network with nodes removal by } \\
\text { coreness in the ascending order }\end{array}$ \\
\hline$\langle C\rangle_{\mathrm{d}}^{\prime}$ & $\begin{array}{c}\text { Cluster coefficient of network with nodes removal by } \\
\text { degree in the ascending order }\end{array}$ \\
\hline$\langle C\rangle_{\mathrm{b}}^{\prime}$ & $\begin{array}{c}\text { Cluster coefficient of network with nodes removal by } \\
\text { betweenness in the ascending order }\end{array}$ \\
\hline$\langle C\rangle_{c}^{\prime}$ & $\begin{array}{c}\text { Cluster coefficient of network with nodes removal by } \\
\text { coreness in the ascending order }\end{array}$ \\
\hline$\sigma$ & Variance \\
\hline
\end{tabular}

are, respectively, listed in the 2 nd column and 5 th of Table 2. In the 2 nd column of Table 2 , the box dimension $\operatorname{Dim}_{d}$ of the original SFnet is 1.6750. After nodes of the SFnet are gradually removed by means of degree in the descending order, the box dimension $\operatorname{Dim}_{d}$ increases; however, the cluster coefficient $\langle C\rangle_{\mathrm{d}}$ of SFnet is more and more small. While $30 \%$ of nodes of SFnet are removed, the box dimension $\operatorname{Dim}_{\mathrm{d}}$ and cluster coefficient $\langle C\rangle_{\mathrm{d}}$ become zero. The results show that after the large-degree nodes are removed, the self-similarity structure of SFnet are gradually destroyed and finally the network structure is completely destroyed. The reason is that the SFnet is constructed by many low-degree nodes, and the large-degree nodes are surrounded by low-degree nodes. When these large-degree nodes are removed, the self-similarity structure is influenced.

The trend of box dimension $\operatorname{Dim}_{\mathrm{d}}$ and cluster coefficient $\langle C\rangle_{\mathrm{d}}$ of SFnet is, respectively, shown in Figures 3(a) and 3(c) after nodes removal. In Figure 3(a), box dimension $\operatorname{Dim}_{d}$ of the SFnet increases gradually while nodes of the highest degree are removed. Finally, the box dimension $\operatorname{Dim}_{d}$ of the network is equal to zero. The fluctuation of box dimension $\operatorname{Dim}_{\mathrm{d}}$ is large, and the variance of dimension is also large, that is, 1.0113. After nodes removal by means of degree in the descending order, the whole network is divided into many independent subnetworks. The shortest distances of 


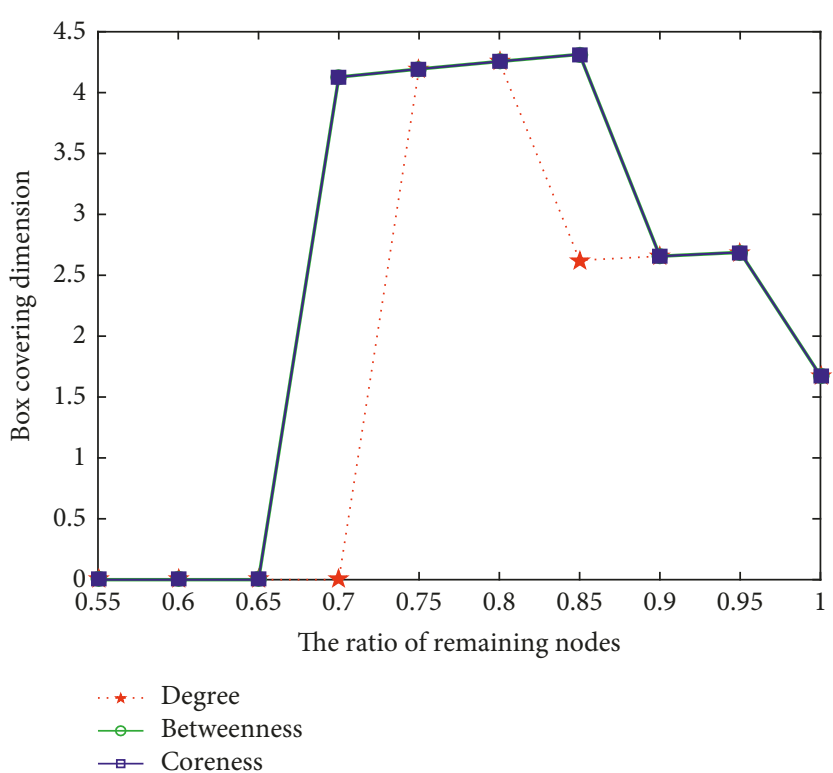

(a)

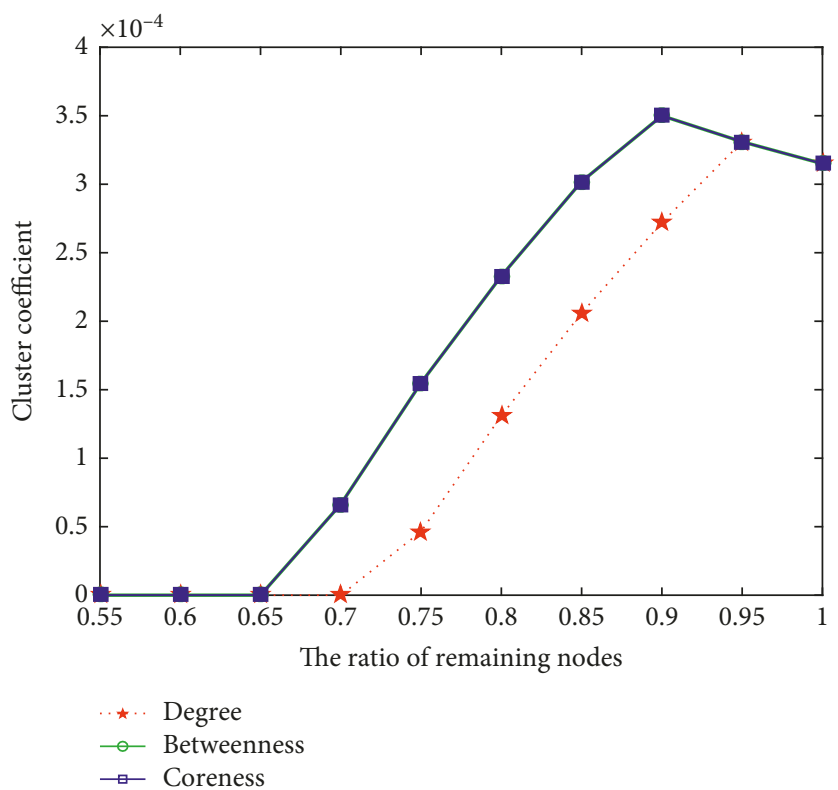

(c)

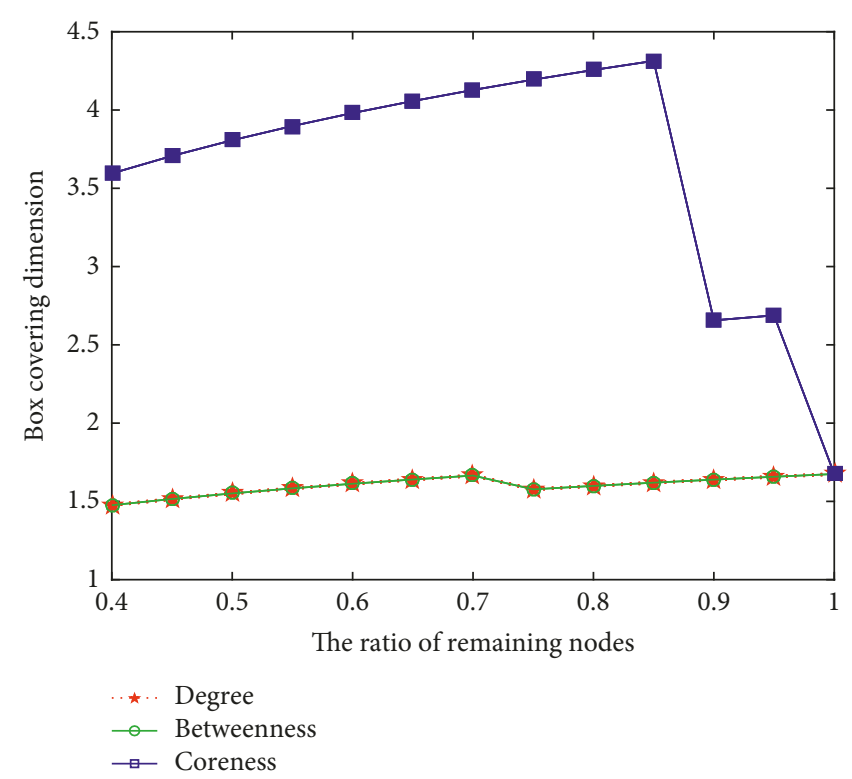

(b)

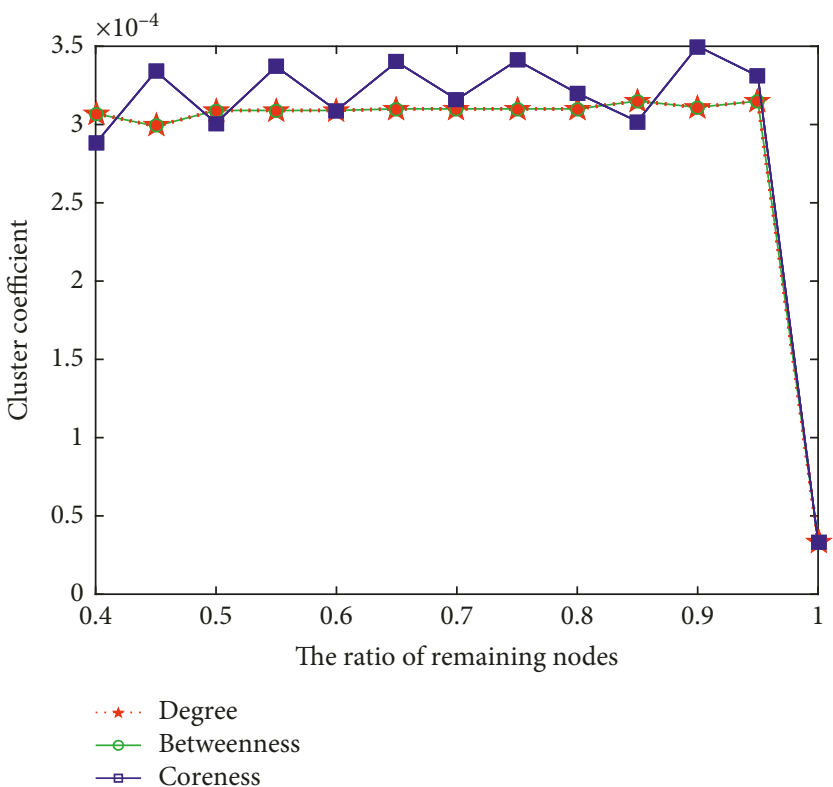

(d)

Figure 3: Changes of box dimension and cluster coefficient after nodes are removed in the ascending and descending order. The $X$-axis represents the number of nodes remaining in the network. (a) The changes of box dimension after nodes removal in the descending order of SFnet. (b) The changes of box dimension after nodes removal in the ascending order of SFnet. (c) Cluster coefficient changes with nodes removal in the descending order of SFnet. (d) Cluster coefficient changes with nodes removal in the ascending order of SFnet.

SFnet are more and more small, the box dimension Dim $_{\mathrm{d}}$ becomes more and more large. In Figure 3(c), the cluster coefficient $\langle C\rangle_{\mathrm{d}}$ decreases. However, when all these central nodes with large degree are removed from the SFnet, the number of boxes need to cover subnetworks is one and the box dimension $\operatorname{Dim}_{d}$ of network is zero (i.e., there is no selfsimilarity characteristic). At this time, the subnetworks are many isolated nodes, and the cluster coefficient is zero, that is, the self-similarity characteristic of SFnet is completely destroyed. The effects of large-degree nodes on self-similarity are high.

When the degree of the SFnet is sorted in the ascending order, the first $5 \%$ of smallest nodes are removed, and the box dimension Dim ${ }_{\mathrm{d}}^{\prime}$ and cluster coefficient $\langle C\rangle_{d}^{\prime}$ of subnetworks are, respectively, listed in the 2 nd column and 5th column of Table 3. In Table 3, the box dimension $\operatorname{Dim}_{\mathrm{d}}^{\prime}$ of original SFnet is 1.6750. After the small-degree nodes are removed, box dimensions $\operatorname{Dim}_{\mathrm{d}}^{\prime}$ of subnetworks have almost 
TABLE 2: Changes of box dimension and cluster coefficient of SFnet with nodes removal in the descending order.

\begin{tabular}{lcccccc}
\hline $\begin{array}{l}\text { Ratio } \\
(\%)\end{array}$ & $\operatorname{Dim}_{\mathrm{d}}$ & $\operatorname{Dim}_{\mathrm{b}}$ & $\operatorname{Dim}_{\mathrm{c}}$ & $\langle C\rangle_{\mathrm{d}}$ & $\langle C\rangle_{\mathrm{b}}$ & $\langle C\rangle_{\mathrm{c}}$ \\
\hline 55 & 0 & 0 & 0 & 0 & 0 & 0 \\
60 & 0 & 0 & 0 & 0 & 0 & 0 \\
65 & 0 & 0 & 0 & 0 & 0 & 0 \\
70 & 0 & 4.1279 & 4.1279 & 0 & $6.64 E-05$ & $6.64 E-05$ \\
75 & 4.1939 & 4.1939 & 4.1939 & $4.65 E-05$ & $1.55 E-04$ & $1.55 E-04$ \\
80 & 4.2557 & 4.2557 & 4.2557 & $1.31 E-04$ & $2.33 E-04$ & $2.33 E-04$ \\
85 & 2.6228 & 4.3138 & 4.3138 & $2.06 E-04$ & $3.02 E-04$ & $3.02 E-04$ \\
90 & 2.6564 & 2.6564 & 2.6564 & $2.72 E-04$ & $3.50 E-04$ & $3.50 E-04$ \\
95 & 2.6881 & 2.6881 & 2.6881 & $3.31 E-04$ & $3.31 E-04$ & $3.31 E-04$ \\
100 & 1.6750 & 1.6750 & 1.6750 & $3.15 E-04$ & $3.15 E-04$ & $3.15 E-04$ \\
$\sigma$ & 1.0113 & 3.4737 & 3.4737 & $1.95 E-08$ & $2.20 E-08$ & $2.20 E-08$ \\
\hline
\end{tabular}

no change. While $30 \%$ of nodes are removed, the box dimension is 1.6651 which is of little change. Even though 60\% of nodes of SFnet are removed, the box dimension Dim ${ }_{d}^{\prime}$ is 1.4758 , that is, the change of box dimension of subnetwork is also small. In the process of nodes removal by means of degree in the ascending order of the SFnet, the fluctuation of box dimension $\operatorname{Dim}_{\mathrm{d}}^{\prime}$ is small and the variance is 0.0036 . The cluster coefficient $\langle C\rangle_{\mathrm{d}}^{\prime}$ increases as nodes of small degrees are removed. Although the cluster coefficient is fluctuating, the fluctuation range is very small. The variance of the clustering coefficient $\langle C\rangle_{\mathrm{d}}^{\prime}$ is $5.88 E-09$, which is close to zero. The trend of box dimension Dim ${ }_{d}^{\prime}$ and cluster coefficient $\langle C\rangle_{d}^{\prime}$ is, respectively, shown in Figures 3(b) and 3(d). In Figure 3(b), the change of box dimension $\operatorname{Dim}_{d}^{\prime}$ of the SFnet is small. The variance of box dimension Dim ${ }_{d}^{\prime}$ change is also small, that is, 0.0036 . When the small-degree nodes are removed, the network is more and more compact. For the SFnet, when the small-degree nodes are removed gradually from the subnetworks, some new small-degree nodes appear. Thus, the changes of self-similarity structure of SFnet are tiny. In Figure 3(d), the cluster coefficient increase is low, which shows that the self-similarity structure of subnetworks is more and more compact. The results show that the influence of small-degree nodes on self-similarity is tiny.

3.2. Influence of Betweenness of Node on Self-Similarity. In Ref. [31], the influence of betweenness on network fractal is discussed by Kitsak et al.; random links are added to network, and network fractal is affected by the crossover length $l^{*}$ and density of random edges $p$. In this section, the effects of betweenness on self-similarity are studied after nodes removal.

The changes of self-similarity of SFnet are discussed by sorting betweenness in the descending order and ascending order after removing nodes gradually. Betweenness is sorted in the descending order, and the first $5 \%$ of maximum betweenness nodes are removed for every rank. After these nodes are removed, box dimension $\operatorname{Dim}_{\mathrm{b}}$ and cluster coefficient $\langle C\rangle_{\mathrm{b}}$ of the subnetworks are, respectively, calculated by using equations (5) and (9). The box dimension $\operatorname{Dim}_{b}$ of the subnetworks is shown in the 3rd column of
Table 2 , and the cluster coefficient $\langle C\rangle_{\mathrm{b}}$ of subnetworks is listed in the 6th column of Table 2.

In Table 2, the change of box dimension $\mathrm{Dim}_{\mathrm{b}}$ is large, from 1.675 on the original network to 4.1279 after $30 \%$ of nodes of SFnet are removed. While more than $30 \%$ of the nodes of SFnet are removed, the box dimension $\operatorname{Dim}_{\mathrm{b}}$ of SFnet becomes zero. As the nodes in the SFnet are removed, the structure of SFnet is changed. The cluster coefficient $\langle C\rangle_{\mathrm{b}}$ of SFnet changes initially from $3.15 E-04$ to $6.64 E-05$ and finally to zero. The network is more and more sparse with nodes removal by betweennesss in the descending order, and the self-similarity structure of SFnet is destroyed.

After the nodes of large betweenness in the SFnet are removed, the trend of the box dimension $\operatorname{Dim}_{\mathrm{b}}$ and cluster coefficient $\langle C\rangle_{\mathrm{b}}$ is shown in Figures 3(a) and 3(c). In Figure 3(a), the changes of box dimension of SFnet are large, and the box dimension Dim $_{b}$ of subnetworks increases first and then decreases to zero. The reason is that some nodes of SFnet with large degree also have large betweenness; the variance change of the box dimension indicates that the box dimension of SFnet varies greatly after the nodes of large betweenness are removed. In Figure 3(c), the cluster coefficient $\langle C\rangle_{\mathrm{b}}$ of SFnet increases first and then decreases. When the nodes with large betweenness are removed, the bridge between nodes is disconnected, the distance required to cover the network increases, and the box dimension decreases. When $30 \%$ of nodes are removed for betweenness in the descending order, the network is divided into independent nodes and box dimension becomes zero. The self-similarity of network is completely destroyed. The results show that large-betweenness nodes have great influence on self-similarity.

By removing nodes in ascending order of betweenness, the changes of box dimension of the subnetwork are shown in the 3rd column of Table 3. In Table 3, the box dimension $\operatorname{Dim}_{b}^{\prime}$ of subnetworks decrease is small. The cluster coefficient $\langle C\rangle_{b}^{\prime}$ of SFnet is listed in the 6th column of Table 3 , and the cluster coefficient $\langle C\rangle_{\mathrm{b}}^{\prime}$ of SFnet also changes to a small extent with small-betweenness nodes removal. The trend of box dimension $\operatorname{Dim}_{b}^{\prime}$ and $\langle C\rangle_{b}^{\prime}$ of SFnet after nodes removal by betweenness ascending order is, respectively, shown in Figures 3 (b) and 3 (d). In Table 3, the box dimension Dim and cluster coefficient $\langle C\rangle_{b}^{\prime}$ of SFnet are the same as removing nodes by means of degree in the ascending order. The reason is that the small-degree nodes also have small betweenness. The results show that small betweenness nodes have less influence on self-similarity.

3.3. Influence of Coreness on Self-Similarity. Some nodes in the center of network have small degree, but these nodes are important [24]. Thus, $\mathrm{k}$ - core is proposed to identify the center of network [47]. $\mathrm{k}$ - core (coreness) has been widely used to identify the importance of nodes and nodes propagation capability $[48,49]$. In this section, with nodes removal by sorting coreness, the influence of coreness on selfsimilarity is discussed.

The coreness of nodes is sorted in the descending order first, and the top 5\% nodes are gradually removed from SFnet with nodes sorting. The changes of box dimension 
TABLE 3: Changes of box dimension and cluster coefficient of SFnet with nodes removal in the ascending order.

\begin{tabular}{lccccr}
\hline Ratio (\%) & $\operatorname{Dim}_{\mathrm{d}}^{\prime}$ & $\operatorname{Dim}_{\mathrm{b}}^{\prime}$ & $\operatorname{Dim}_{\mathrm{c}}^{\prime}$ & $\langle C\rangle_{\mathrm{d}}^{\prime}$ & $\langle C\rangle_{\mathrm{b}}^{\prime}$ \\
\hline 40 & 1.4758 & 1.4758 & 3.5950 & $3.07 E-04$ & $3.07 E-04$ \\
45 & 1.5155 & 1.5155 & 3.7067 & $2.99 E-04$ & $2.99 E-04$ \\
50 & 1.5510 & 1.5510 & 3.8069 & $3.09 E-04$ & $3.09 E-04$ \\
55 & 1.5833 & 1.5833 & 3.8977 & $3.09 E-04$ & $3.09 E-04$ \\
60 & 1.6128 & 1.6128 & 3.9807 & $3.09 E-04$ & $3.09 E-04$ \\
65 & 1.6399 & 1.6399 & 4.0571 & $3.10 E-04$ & $3.10 E-04$ \\
70 & 1.6651 & 1.6651 & 4.1279 & $3.10 E-04$ & $3.10 E-04$ \\
75 & 1.5770 & 1.5770 & 4.1939 & $3.10 E-04$ & $3.10 E-04$ \\
80 & 1.5990 & 1.5990 & 4.2557 & $3.10 E-04$ & $3.10 E-04$ \\
85 & 1.6196 & 1.6196 & 4.3138 & $3.15 E-04$ & $3.15 E-04$ \\
90 & 1.6391 & 1.6391 & 2.6564 & $3.11 E-04$ & $3.11 E-04$ \\
95 & 1.6575 & 1.6575 & 2.6881 & $3.15 E-04$ & $3.16 E-04$ \\
100 & 1.6750 & 1.6750 & 1.6750 & $3.35 E-05$ & $3.15 E-04$ \\
$\sigma$ & 0.0036 & 0.0036 & 0.6245 & $5.88 E-09$ & $3.35 E-04$ \\
\end{tabular}

$\operatorname{Dim}_{c}$ of subnetworks are calculated, which are shown in the 4 th column of Table 2; the trend of box dimension $\operatorname{Dim}_{c}$ change is shown in Figure 3(a). In addition, the changes of cluster coefficient $\langle C\rangle_{\mathrm{c}}$ are calculated with nodes removal, the results are shown in the 7 th column of Table 2, and Figure 3(c) shows the trend of cluster coefficient $\langle C\rangle_{\mathrm{c}}$.

In Table 3, the changes box dimension $\operatorname{Dim}_{c}$ of SFnet is large. Because degree, betweenness, and coreness are the same for most of the nodes in the SFnet, the change of coreness is similar with nodes removal by means of betweenness in the descending order. With nodes removal, box dimension $\operatorname{Dim}_{\mathrm{c}}$ of subnetworks gradually increases. While $30 \%$ of nodes are removed, box dimension $\operatorname{Dim}_{c}$ suddenly decreases to zero. In Figure 3(c), the cluster coefficient gradually decreases with nodes removal, which shows that the self-similarity structure of SFnet is destroyed with large-coreness nodes removal. When the cluster coefficient is zero, the self-similarity structure of network is completely destroyed.

In Table 3, the variance of box dimension is large. After removing nodes by coreness in the ascending order, the change of box dimension of SFnet is large. The trend of box dimension $\operatorname{Dim}_{c}^{\prime}$ is shown in Figure 3(b). In Figure 3(b), box dimension $\operatorname{Dim}_{\mathrm{c}}^{\prime}$ increases first and then decreases. In Figure $3(\mathrm{~d})$, the trend of cluster coefficient $\langle C\rangle_{\mathrm{c}}^{\prime}$ of SFnet increases; that is, the network SFnet is compact with nodes removal. The results show that small-coreness nodes have influence on self-similarity of SFnet.

In Ref. [28], the Jaccard similarity coefficient is introduced to measure the neighborhood similarity, which is used to identify the importance of network nodes, and the method is effective [28]. For the proposed method, the center nodes are important and have large influence on the self-similarity of SFnet. The results are the same as judging the importance of nodes of SFnet with neighborhood similarity.

From the above information, it is known that the selfsimilarity of SFnet is influenced by degree, betweenness, and coreness. The effects on constructed SFnet are great, especially for descending order nodes removal. The box dimension fluctuation is large. The detailed box dimension and cluster coefficient variance are shown in the last row of
Tables 2 and 3, and the box dimension change of SFnet is shown in Figures 3(a) and 3(b). In Figure 3, the box dimension of SFnet greatly fluctuates, and degree, betweenness, and coreness are similar. In addition, in the nodes removal process, there is a critical point of dimension change, that is, about $30 \%$ of nodes are removed. The selfsimilarity structure of network changes greatly, and the changes of cluster coefficient are also large. Large degree and betweenness have great effects on self-similarity.

\section{Verification to Real Networks}

In this section, two self-similarity networks including a USAir network and the YPI network are used to illustrate the influence of nodes removal on self-similarity.

4.1. USAir Network. The USAir network has been studied by many scholars, and the self-similarity of the network was proved [9, 12, 50]. The USAir network has 332 nodes and 2126 edges. These nodes represent position, and edges represent the air lines of every two positions [51].

When degree, betweenness, and coreness of nodes of the USAir network are sorted in the descending order, the first $5 \%$ of the biggest nodes are gradually removed, and using equation (9), box dimension of the network is calculated. The results of box dimension are shown in Table 4 .

In the 2 nd and 3 rd columns of Table 4, the range of variation of the box dimension is large. Dim $_{d}$ changes from 2.9415 to 7.4838 , and $\operatorname{Dim}_{\mathrm{b}}$ also changes from 2.9415 to 7.4838. The variances of $\operatorname{Dim}_{d}$ and $\operatorname{Dim}_{b}$ are 5.6475 and 3.7255 , respectively. In the last row of Table 4 , the variance of box dimension is large which is more than 3 . The changes of fractal dimension are large with nodes removal by degree and betweenness in the descending order because these nodes have great information transfer and influence on selfsimilarity. While these nodes are removed, the structure of network is changed and self-similarity structure is destroyed. In the 5 th and 6 th column of Table 4 , the cluster coefficient decreases with the large degree and betweenness nodes removal; that is, the structure of self-similarity of network is 
TABLE 4: Changes of box dimension and cluster coefficient of USAir network with nodes removal in the descending order.

\begin{tabular}{lccccr}
\hline Ratio (\%) & $\operatorname{Dim}_{\mathrm{d}}$ & $\operatorname{Dim}_{\mathrm{b}}$ & $\operatorname{Dim}_{\mathrm{c}}$ & $\langle C\rangle_{\mathrm{d}}$ & $\langle C\rangle_{\mathrm{b}}$ \\
\hline 55 & 7.4838 & 7.4838 & 1.7798 & $4.47 E-06$ & 0.0168 \\
60 & 7.6147 & 4.9993 & 1.3400 & $1.22 E-05$ & 0.0306 \\
65 & 2.2754 & 5.0798 & 1.0329 & $1.60 E-05$ & 0.0753 \\
70 & 2.3086 & 3.1700 & 1.1442 & 0.0261 & 0.0673 \\
75 & 1.9434 & 2.4399 & 1.1553 & 0.0567 & 0.1119 \\
80 & 1.5288 & 1.6070 & 1.2156 & 0.1159 & 0.1657 \\
85 & 1.4380 & 1.7678 & 1.2281 & 0.1918 & 0.1828 \\
90 & 1.6406 & 1.8014 & 2.1167 & 0.2794 & 0.2246 \\
95 & 2.0042 & 2.0214 & 2.4643 & 0.3843 & 0.2929 \\
100 & 2.9415 & 2.9415 & 2.9415 & 0.6252 & 0.3626 \\
$\sigma$ & 5.6475 & 3.7255 & 0.4343 & 0.0432 & 0.2693 \\
\hline
\end{tabular}

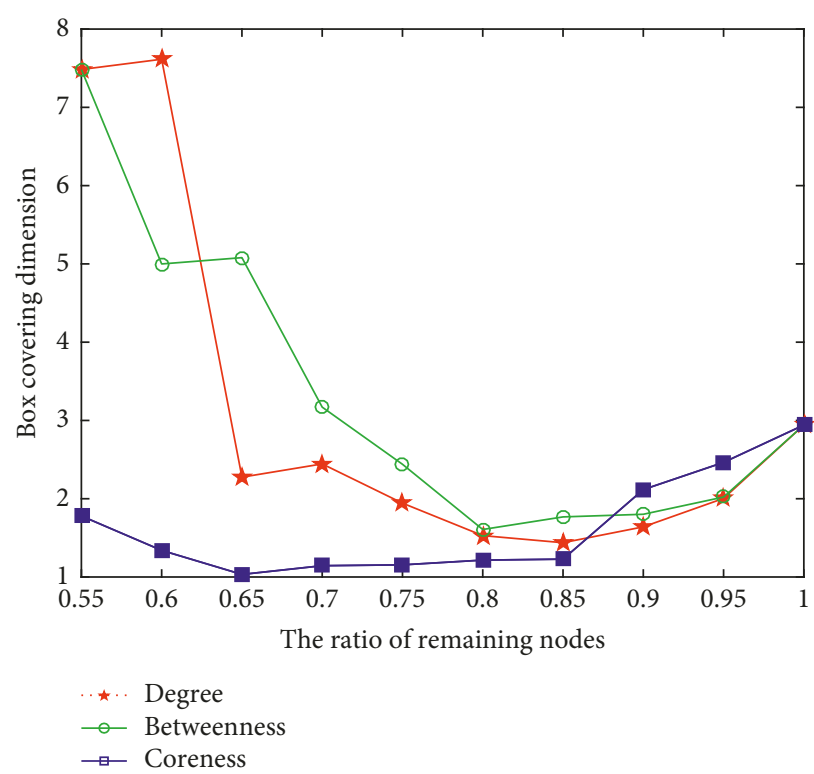

(a)

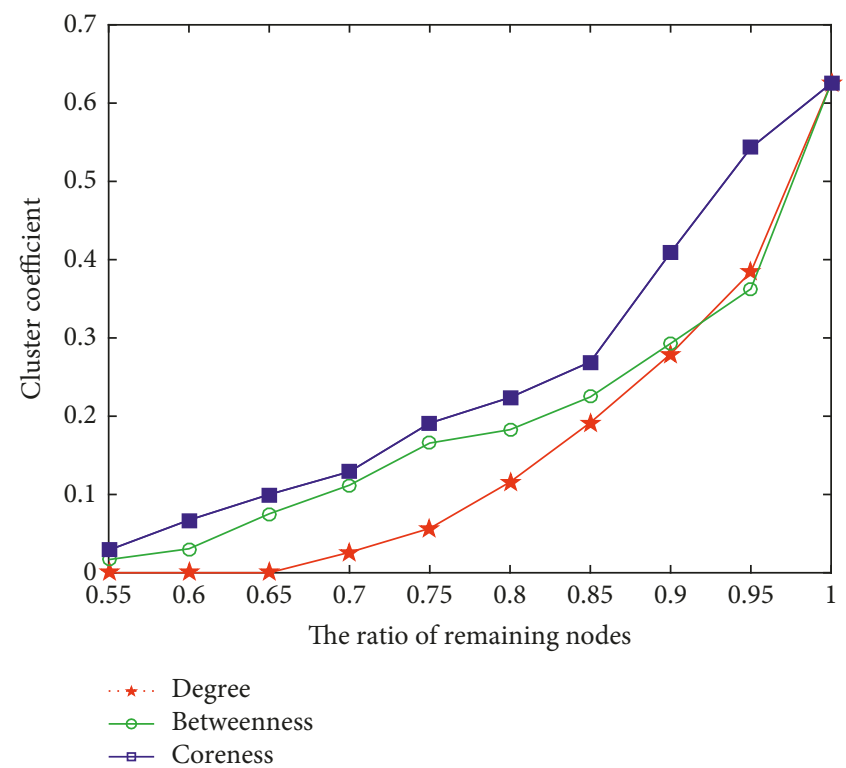

(c)

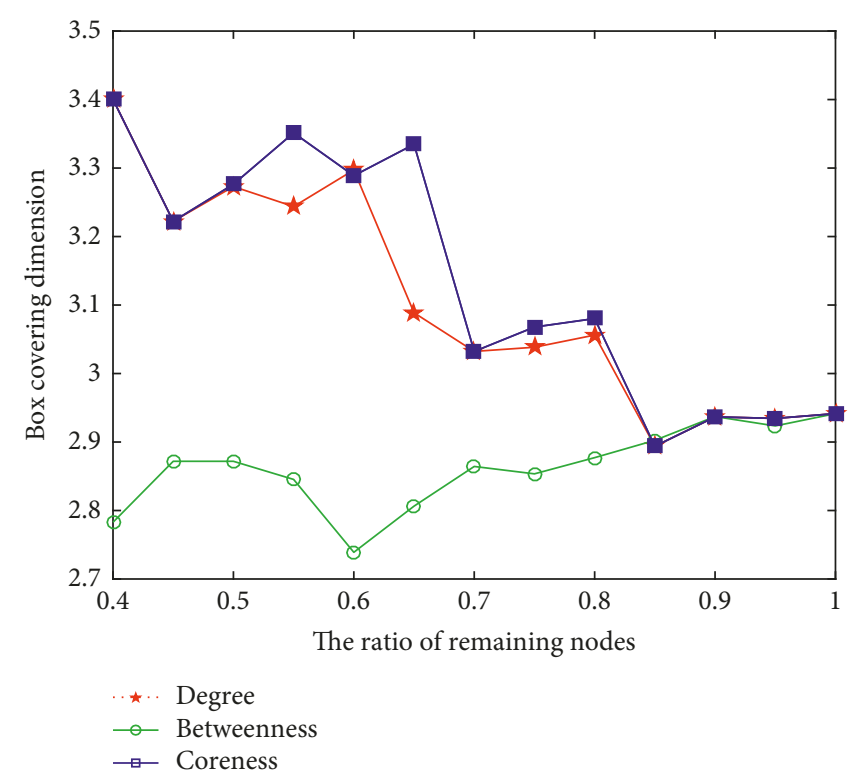

(b)

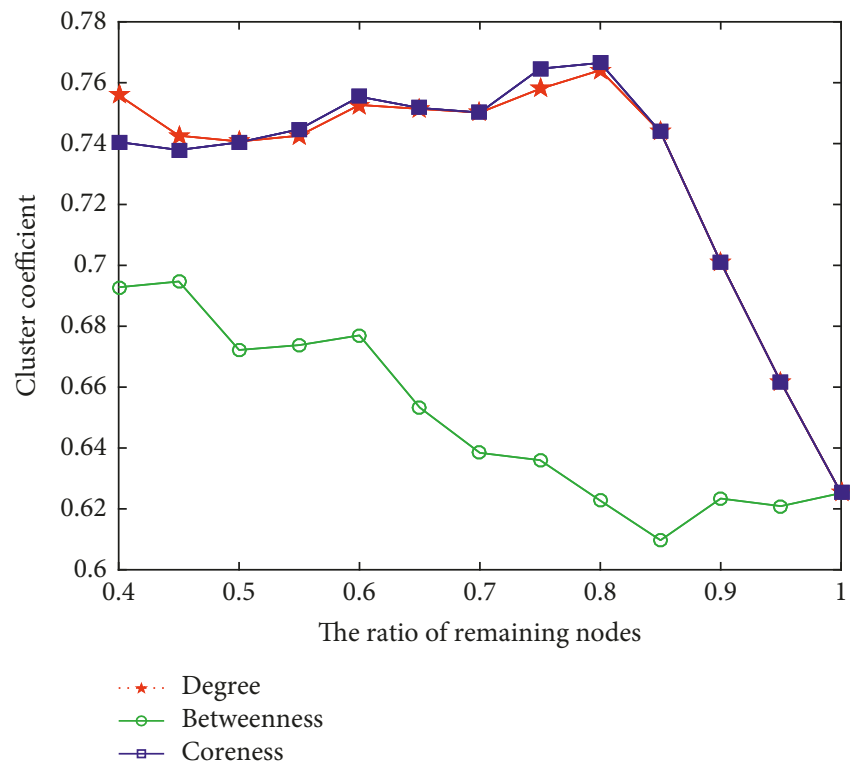

(d)

FIGURE 4: Changes of box dimension and cluster coefficient of USAir network, after nodes are removed by sorting. The $X$-axis represents the number of nodes remaining in the network. (a) Nodes are removed by degree, betweenness, and coreness in the descending order. $5 \%$ of the biggest nodes are removed from the network in each rank, and the dimension of remaining network is calculated. (b) Nodes are removed by coreness in the ascending order. 5\% of the smallest nodes are removed from the network in each rank, and the dimension of remaining network is calculated. (c) The changes of cluster coefficient after nodes are removed by degree, betweenness, and coreness in the descending order. (d) The changes of cluster coefficient after nodes are removed by degree, betweenness, and coreness in the ascending order. 
TABLE 5: Changes of box dimension and cluster coefficient of USAir network with nodes removal by ascending order.

\begin{tabular}{|c|c|c|c|c|c|c|}
\hline Ratio (\%) & $\operatorname{Dim}_{\mathrm{d}}^{\prime}$ & $\operatorname{Dim}_{b}^{\prime}$ & $\operatorname{Dim}_{c}^{\prime}$ & $\langle C\rangle_{\mathrm{d}}^{\prime}$ & $\langle C\rangle_{\mathrm{b}}^{\prime}$ & $\langle C\rangle_{\mathrm{c}}^{\prime}$ \\
\hline 40 & 3.4002 & 2.7828 & 3.4002 & 0.7559 & 0.6928 & 0.7405 \\
\hline 45 & 3.2222 & 2.8719 & 3.2222 & 0.7426 & 0.6947 & 0.7379 \\
\hline 50 & 3.2730 & 2.8718 & 3.2763 & 0.7407 & 0.6722 & 0.7404 \\
\hline 55 & 3.2438 & 2.8454 & 3.3514 & 0.7426 & 0.6738 & 0.7447 \\
\hline 60 & 3.2972 & 2.7390 & 3.2889 & 0.7527 & 0.6770 & 0.7554 \\
\hline 65 & 3.0887 & 2.8064 & 3.3347 & 0.7514 & 0.6533 & 0.7517 \\
\hline 70 & 3.0323 & 2.8643 & 3.0323 & 0.7502 & 0.6384 & 0.7502 \\
\hline 75 & 3.0384 & 2.8535 & 3.0678 & 0.7580 & 0.6360 & 0.7646 \\
\hline 80 & 3.0561 & 2.8772 & 3.0803 & 0.7641 & 0.6227 & 0.7665 \\
\hline 85 & 2.8938 & 2.9025 & 2.8938 & 0.7438 & 0.6097 & 0.7438 \\
\hline 90 & 2.9367 & 2.9372 & 2.9367 & 0.7010 & 0.6234 & 0.7010 \\
\hline 95 & 2.9345 & 2.9235 & 2.9345 & 0.6616 & 0.6209 & 0.6616 \\
\hline 100 & 2.9415 & 2.9415 & 2.9415 & 0.6252 & 0.6252 & 0.6252 \\
\hline$\sigma$ & 0.0272 & 0.0036 & 0.0335 & 0.0018 & 0.0009 & 0.0018 \\
\hline
\end{tabular}

TABLE 6: Box dimension and cluster coefficient of YPI network with nodes removal in the descending order.

\begin{tabular}{lccccc}
\hline Ratio (\%) & $\operatorname{Dim}_{\mathrm{d}}$ & $\operatorname{Dim}_{\mathrm{b}}$ & $\operatorname{Dim}_{\mathrm{c}}$ & $\langle C\rangle_{\mathrm{d}}$ & $\langle C\rangle_{\mathrm{b}}$ \\
\hline 55 & 0 & 10.3432 & 2.8497 & 0 & $4.85 E-05$ \\
60 & 10.4686 & 10.4686 & 2.9111 & $2.39 E-05$ & $4.72 E-05$ \\
65 & 4.2835 & 10.5840 & 2.9417 & $6.56 E-05$ & $5.21 E-05$ \\
70 & 4.3273 & 5.3386 & 1.7971 & $6.43 E-05$ & 0.0048 \\
75 & 1.5414 & 3.3922 & 1.7485 & $9.25 E-05$ & 0.0424 \\
80 & 1.1784 & 2.0578 & 1.8949 & 0.0009 & 0.0255 \\
85 & 1.6934 & 1.3925 & 2.3363 & 0.0191 & 0.0429 \\
90 & 2.2002 & 1.8067 & 2.4586 & 0.0479 & 0.0573 \\
95 & 2.5309 & 2.3848 & 2.6912 & 0.0870 & 0.0748 \\
100 & 2.7397 & 2.7397 & 2.7397 & 0.1303 & 0.0986 \\
$\sigma$ & 8.4641 & 15.1252 & 0.2216 & 0.0021 & 0.1303 \\
\hline
\end{tabular}

destroyed. In Figure 4(a), the influence of nodes of large degree and betweenness on box dimension of USAir network is large. There is a critical point (i.e., about $30 \%$ of nodes are removed for degree or betweenness descending order), and box dimension suddenly greatly changes. The changes of box dimension are small with nodes removal by coreness in the descending order; the variance of box dimension $\operatorname{Dim}_{\mathrm{c}}$ is 0.4343 , which shows that coreness has small effect on box dimension of USAir network when nodes are removed in the descending order. In Figure 4(c), the range of fluctuations of cluster coefficient $\langle C\rangle_{d}$ and $\langle C\rangle_{b}$ is large. The results show that large degree and betweenness have great influence on self-similarity of USAir network.

When nodes are removed by degree, betweenness, and coreness in the ascending order, the results of box dimension and cluster coefficient are shown in Table 5. The ranges of fluctuation results are small, in the last row of Table 5; the variance of both of them is less than 0.1 even though $60 \%$ of nodes are removed. While these small degree, betweenness, and coreness nodes are removed, the structure of USAir network is more and more compact. In Figure 4(b), box dimension increases with nodes removal in the ascending order and cluster coefficient also gradually increases as shown in Figure 4(d). The results show that after removing nodes by degree, betweenness, and coreness in the ascending order, the influence these nodes have on self-similarity is small.
For the USAir network, nodes 118, 261, 255, and 152 have large degree and betweenness. In fact, the degree and betweenness of node 118 (i.e., Chicago O Hare Intl) are the biggest. Until 1998, Chicago O Hare was the busiest airport in the world in terms of number of passengers (https://en. wikipedia.org/wiki/O\%\27Hare_International_Airport) [39]. After the nodes are removed, the self-similarity structure is destroyed. That is, this airport is very important and has a great impact on the structure of the USAir network. The practical significance of this airport is very great, and people should pay enough attention to it and protect it effectively.

In summary, nodes of large degree or betweenness have great influence on self-similarity with gradual removal of nodes. Small degree and betweenness nodes have tiny effects on self-similarity of USAir network. In addition, there is a critical point, when about $30 \%$ nodes are removed for degree or betweenness in the descending order, and the self-similarity structure is almost completely destroyed. The influence of coreness on self-similarity is small.

4.2. YPI Network. As a biological network, the YPI network is closely related to our life, so it has been widely studied by many scholars and its self-similarity has also been confirmed $[7,15,50,52]$. The YPI network consists of 2361 nodes and 7182 edges. These nodes represent the name of protein, and 


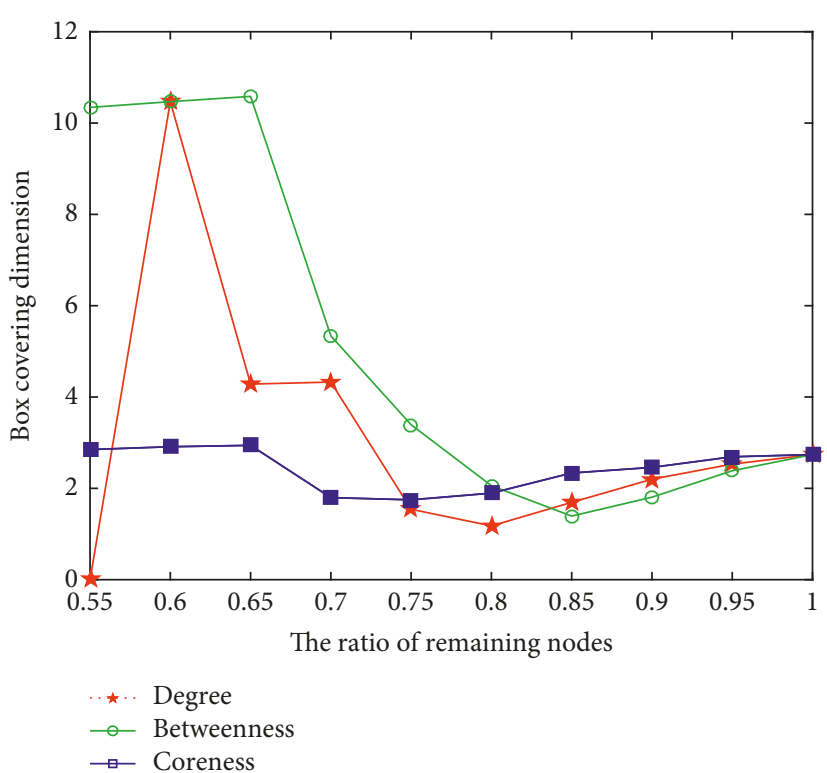

(a)

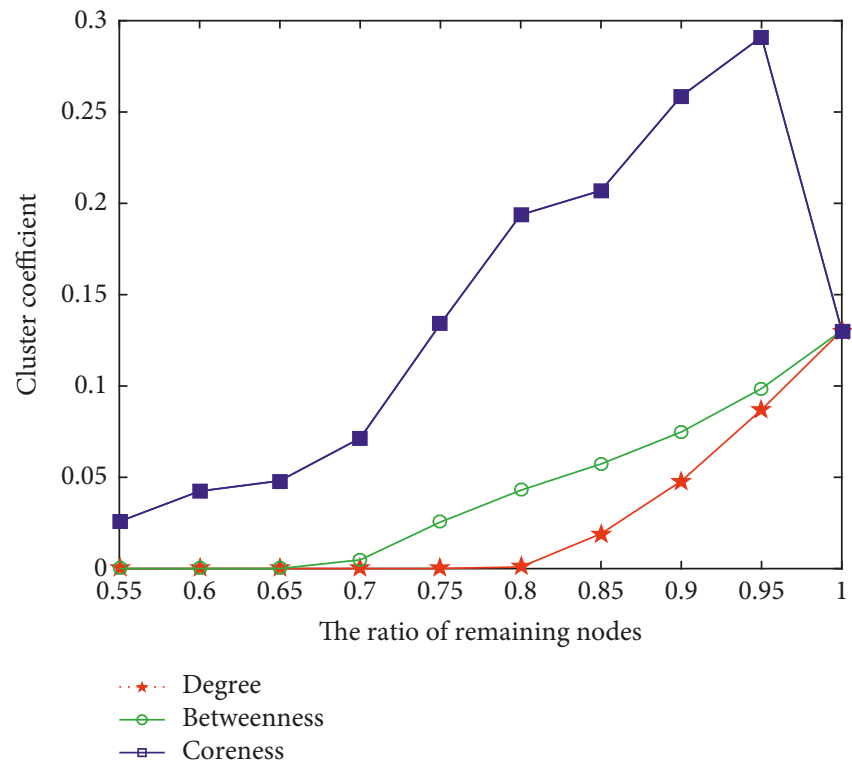

(c)

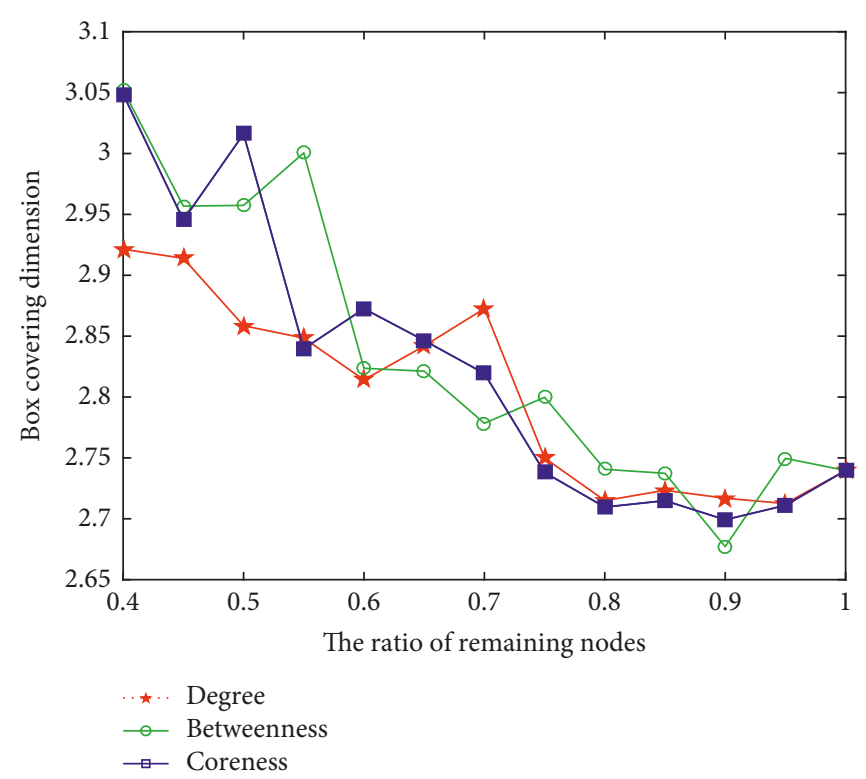

(b)

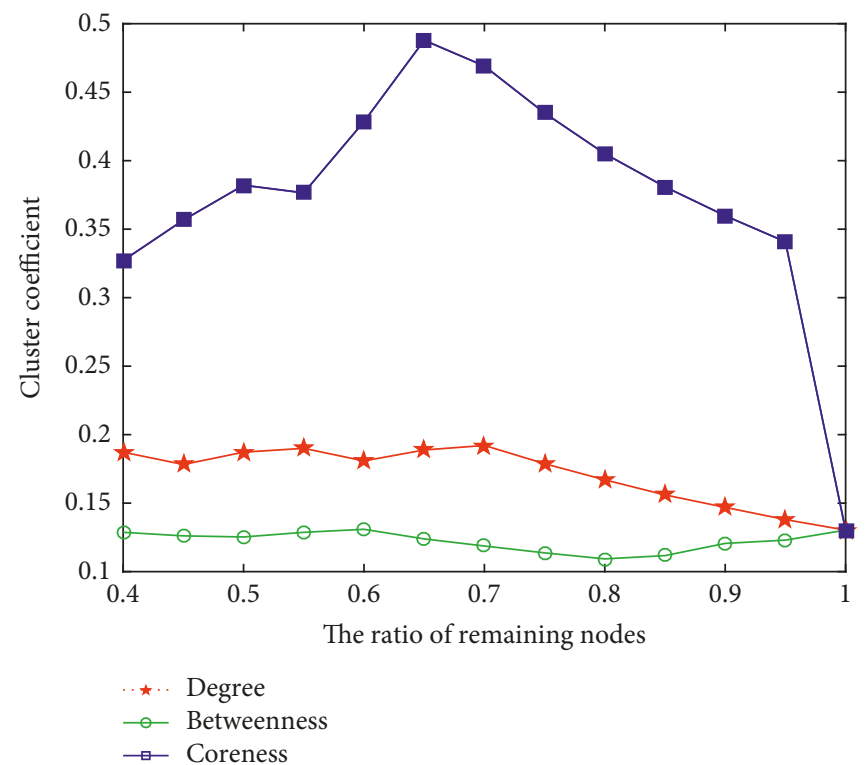

(d)

Figure 5: Changes of box dimension and cluster coefficient of YPI network, after nodes are removed by sorting. The $X$-axis represents the number of nodes remaining in the network. (a) Nodes are removed by degree, betweenness, and coreness in the descending order. The first $5 \%$ of the biggest nodes are removed from the network in each rank, and the dimension of remaining network is calculated. (b) Nodes are removed by coreness in the ascending order. The first $5 \%$ of the smallest nodes are removed from the network, and the dimension of remaining network is calculated. (c) The changes of cluster coefficient after nodes are removed by degree, betweenness, and coreness in the descending order. (d) The changes of cluster coefficient after nodes are removed by degree, betweenness, and coreness in the ascending order.

edges represent two proteins which have some interaction or relation [53].

The nodes are sorted in the descending order of degree, betweenness, and coreness, and the first $5 \%$ of the biggest nodes are gradually removed with nodes sorting. Using equation (9), box dimension are calculated. The results of box dimension are shown in Table 6. In the 2nd and 3rd columns of Table 6 , the change of box dimension is large, and the fractal dimensions have all increased from 2.7397 to more than 10. The cluster coefficient also has great change as shown in the 5th and 6th columns of Table 6 . The results show that the nodes of large degree or betweenness have large influence on self-similarity.

The trend of box dimension is shown in Figure 5(a). In the figure, the changes of box dimension are large with nodes removal by degree and betweenness in the descending order, and the fluctuation range of box dimension is large. When about $30 \%$ of nodes are removed 
TABLE 7: Box dimension and cluster coefficient of YPI network with nodes removal in the ascending order.

\begin{tabular}{lcccccc}
\hline Ratio (\%) & $\operatorname{Dim}_{\mathrm{d}}^{\prime}$ & $\operatorname{Dim}_{b}^{\prime}$ & $\operatorname{Dim}_{\mathrm{c}}^{\prime}$ & $\langle C\rangle_{\mathrm{d}}^{\prime}$ & $\langle C\rangle_{\mathrm{b}}^{\prime}$ & $\langle C\rangle_{\mathrm{c}}^{\prime}$ \\
\hline 40 & 2.9214 & 3.0523 & 3.0487 & 0.1872 & 0.1287 & 0.3271 \\
45 & 2.9139 & 2.9568 & 2.9453 & 0.1782 & 0.1261 & 0.3566 \\
50 & 2.8586 & 2.9574 & 3.0161 & 0.1872 & 0.1253 & 0.3821 \\
55 & 2.8484 & 3.0005 & 2.8396 & 0.1900 & 0.1287 & 0.3765 \\
60 & 2.8141 & 2.8236 & 2.8725 & 0.1808 & 0.1309 & 0.4285 \\
65 & 2.8419 & 2.8212 & 2.8465 & 0.1888 & 0.1239 & 0.4879 \\
70 & 2.8726 & 2.7784 & 2.8193 & 0.1921 & 0.1187 & 0.4691 \\
75 & 2.7499 & 2.7998 & 2.7381 & 0.1788 & 0.1137 & 0.4348 \\
80 & 2.7150 & 2.7406 & 2.7096 & 0.1670 & 0.1093 & 0.4051 \\
85 & 2.7234 & 2.7374 & 2.7149 & 0.1560 & 0.1117 & 0.3809 \\
90 & 2.7169 & 2.6772 & 2.6992 & 0.1469 & 0.1206 & 0.3598 \\
95 & 2.7125 & 2.7496 & 2.7110 & 0.1380 & 0.1230 & 0.3408 \\
100 & 2.7397 & 2.7397 & 2.7397 & 0.1303 & 0.1303 & 0.1303 \\
$\sigma$ & 0.0062 & 0.0141 & 0.0144 & 0.0005 & $5.12 E-05$ & 0.0077 \\
\hline
\end{tabular}

for degree or betweenness in the descending order, there is also a critical point; i.e., box dimension change is large, where the network is divided into many parts and the selfsimilarity structure of network is destroyed. The variance of box dimension is more than 8 after nodes removal by degree and betweenness in the descending order. Large degree and betweenness nodes have great influence on selfsimilarity of YPI network. Form Figure 5(c), the cluster coefficient decreases which shows that the structure of YPI network is more and more sparse. Moreover, form Figure 5(a), the changes of box dimension is small with nodes removal by coreness in the descending order; the influence of coreness on box dimension of YPI network is small after nodes removal.

While nodes are removed by degree, betweenness, and coreness in the ascending order, the box dimension and cluster coefficient results are listed in Table 7 . In the 5 th to 7 th column of Table 7 , the changes of cluster coefficient increase; the results show that when nodes are removed, the self-similarity structure of network does not change too much, and the network is compact. The results of box dimension fluctuation are shown in the last row of Table 7; the changes of variance are no more than 0.1 . The trend of box dimension is shown in Figure 5(b); the changes of box dimension and cluster coefficient are small; the changes of variance are no more than 0.1 . The results show that the nodes of small degree, betweenness, and coreness have little influence on self-similarity.

The degree and betweenness of nodes 566, 209, 302, 1443,784 , and 147 are larger; after these nodes are removed, the network self-similarity structure is severely damaged. The results show that these proteins play an important role in biological reactions, and these proteins should be focused on in biological research and disease prevention research. In conclusion, the nodes with large degree and betweenness have great influence on self-similarity. The effects of small degree and betweenness nodes on self-similarity are small. When about $30 \%$ of nodes are removed by means of degree or betweenness in the descending order, the self-similarity structure of YPI network is almost completely destroyed.
The coreness has small effects on self-similarity of YPI network.

\section{Conclusion}

In this paper, the effects of three statistical variables on selfsimilarity of network are studied. Not only the influence of degree, betweenness, and coreness of the nodes removal in the descending order on self-similarity are considered but also the effects of ascending order nodes removal are considered. A constructed self-similarity network and two real networks (i.e., a USAir network and the YPI network) are applied to numerical simulation. The results show that nodes with large degree and betweenness have great influence on self-similarity of the network. The cluster coefficient of the network is used to judge the structure change in the process of nodes removal, and the influence of coreness on selfsimilarity is small.

Degree, betweenness, and coreness of nodes are, respectively, sorted in the descending and ascending order. The first $5 \%$ of the biggest nodes are removed each time the box dimension is calculated by the box covering algorithm. Moreover, the box dimensions of original networks and subnetworks are compared, and the variance of box dimension and cluster coefficient of subnetworks are also calculated with nodes removal. The results show that large degree and betweenness have great influence on self-similarity of the network for descending order nodes removal, and the influence of coreness is small. In the nodes removal process, network structure is destroyed when about $30 \%$ of nodes are removed by degree or betweenness in the descending order, and the variance of dimension change is large which is more than 1 . When nodes are removed in the ascending order, self-similarity change is small, and the range of variance is tiny which is no more than 0.1 . The research of this paper is of great help for the exploration of the origin of the properties of self-similarity.

\section{Data Availability}

The data of USAir network and the YPI network of this paper can also be found in http://vlado.fmf.uni-lj.si/pub/ networks/data/mix/usair97.net and http://vlado.fmf.uni-lj. si/pub/networks/data/bio/yeast/yeast.htm, respectively.

\section{Conflicts of Interest}

The authors declare that there are no conflicts of interest regarding the publication of this paper.

\section{Authors' Contributions}

All authors read and approved the manuscript.

\section{Acknowledgments}

This work was partially supported by the National Natural Science Foundation of China (Grant nos. 61763009 and 61364030). 


\section{References}

[1] B. Mandelbrot, "How long is the coast of Britain? Statistical self-similarity and fractional dimension," Science, vol. 156, no. 3775 , pp. 636-638, 1967.

[2] R. R. Brook, "A study of self-similarity in turbulence measured at an urban site," Journal of Applied Meteorology, vol. 13, no. 7, pp. 767-770, 1974.

[3] Y. Sawada, S. Ohta, M. Yamazaki, and H. Honjo, "Selfsimilarity and a phase-transition-like behavior of a random growing structure governed by a nonequilibrium parameter," Physical Review A, vol. 26, pp. 272-281, 1982.

[4] J. Hutchinson, "Fractals and self similarity," Indiana University Mathematics Journal, vol. 30, no. 5, pp. 713-747, 1981.

[5] M. Imran, A. Q. Baig, and W. Khalid, "On topological indices of fractal and cayley tree type dendrimers," Discrete Dynamics in Nature and Society, vol. 2018, Article ID 2684984, 11 pages, 2018.

[6] M. E. Crovella and A. Bestavros, "Self-similarity in world wide web traffic: evidence and possible causes," IEEE/ACM Transactions on Networking, vol. 5, no. 6, pp. 835-846, 1997.

[7] C. Song, S. Havlin, and H. A. Makse, "Self-similarity of complex networks," Nature, vol. 433, no. 7024, pp. 392-395, 2005.

[8] C. Song, L. K. Gallos, S. Havlin, and H. A. Makse, "How to calculate the fractal dimension of a complex network: the box covering algorithm," Journal of Statistical Mechanics Theory and Experiment, vol. 2007, pp. 297-316, 2007.

[9] D. J. Wei, Q. Liu, H. X. Zhang, Y. Hu, Y. Deng, and S. Mahadevan, "Box-covering algorithm for fractal dimension of weighted networks," Scientific Reports, vol. 3, no. 1, pp. 3049-3057, 2013.

[10] Y. Song, J. Liu, Z. Yu, and B. Li, "Multifractal analysis of weighted networks by a modified sandbox algorithm," Scientific Reports, vol. 5, no. 1, pp. 17628-17637, 2015.

[11] D. Wei, B. Wei, Y. Hu, H. Zhang, and Y. Deng, "A new information dimension of complex networks," Physics Letters A, vol. 378, no. 16-17, pp. 1091-1094, 2014.

[12] T. Wen and W. Jiang, "An information dimension of weighted complex networks," Physica A: Statistical Mechanics and Its Applications, vol. 501, pp. 388-399, 2018.

[13] M. Dai, Y. Sun, S. Shao, L. Xi, and W. Su, "Modified box dimension and average weighted receiving time on the weighted fractal networks," Scientific Reports, vol. 5, no. 1, pp. 18210-18209, 2015.

[14] D. Li, K. Kosmidis, A. Bunde, and S. Havlin, "Dimension of spatially embedded networks," Nature Physics, vol. 7, pp. 481-484, 2011.

[15] D. Wei, B. Wei, H. Zhang, C. Gao, and Y. Deng, "A generalized volume dimension of complex networks," Journal of Statistical Mechanics: Theory and Experiment, vol. 2014, no. 10, Article ID P10039, 2014.

[16] E. Rosenberg, "Maximal entropy coverings and the information dimension of a complex network," Physics Letters A, vol. 381 , no. 6 , pp. $574-580,2017$.

[17] Z. Shao, S. Sheikholeslami, P. Wu, and J. Liu, "The metric dimension of some generalized petersen graphs," Discrete Dynamics in Nature and Society, vol. 2018, Article ID 4351958, 10 pages, 2018.

[18] C. Song, S. Havlin, and H. A. Makse, "Origins of fractality in the growth of complex networks," Nature Physics, vol. 2, no. 4, pp. 275-281, 2006.

[19] L. K. Gallos, C. Song, and H. A. Makse, "A review of fractality and self-similarity in complex networks," Physica A: Statistical
Mechanics and Its Applications, vol. 386, no. 2, pp. 686-691, 2007.

[20] G. Cao, Y. Shi, and Q. Li, "Structure characteristics of the international stock market complex network in the perspective of whole and part," Discrete Dynamics in Nature and Society, vol. 2017, Article ID 9731219, 11 pages, 2017.

[21] A. Barrat, M. Barthelemy, R. Pastor-Satorras, and A. Vespignani, "The architecture of complex weighted networks," Proceedings of the National Academy of Sciences, vol. 101, no. 11, pp. 3747-3752, 2004.

[22] E. Estrada, "Quantifying network heterogeneity," Physical Review E, vol. 82, no. 6, pp. 66102-66109, 2010.

[23] L. Lü, D. Chen, X.-L. Ren, Q.-M. Zhang, Y.-C. Zhang, and T. Zhou, "Vital nodes identification in complex networks," Physics Reports, vol. 650, pp. 1-63, 2016.

[24] X. Ren and L. L, “网络重要节点排序方法综述,” Chinese Science Bulletin, vol. 59, no. 13, pp. 1175-1197, 2014.

[25] R. Yi Run, L. Song Yang, W. Jun De, and B. Liang, "Ranking node importance in large-scale complex network: from a perspective of local abnormal links," in Proceedings of the 2017 3rd International Conference on Big Data Computing and Communications (BIGCOM), pp. 350-353, Chengdu, China, August 2017.

[26] Y. Yang, G. Xie, and J. Xie, "Mining important nodes in directed weighted complex networks," Discrete Dynamics in Nature and Society, vol. 2017, Article ID 9741824, 7 pages, 2017.

[27] Z. Yi, X. Wu, and F. Li, "Ranking spreaders in complex networks based on the most influential neighbors," Discrete Dynamics in Nature and Society, vol. 2018, Article ID 3649079, 6 pages, 2018.

[28] R. Yi Run, L. Song Yang, W. Jun De, B. Liang, and C. Li Dong, "Node importance measurement based on neighborhood similarity in complex network," Acta Physica Sinica, vol. 66, pp. 38902-38911, 2017.

[29] M. P. Joy, A. Brock, D. E. Ingber, and S. Huang, "High-betweenness proteins in the yeast protein interaction network," Journal of Biomedicine and Biotechnology, vol. 2005, no. 2, pp. 96-103, 2005.

[30] Y. R. Ruan, S. Y. Lao, Y. D. Xiao, J. D. Wang, and L. Bai, "Identifying influence of nodes in complex networks with coreness centrality: decreasing the impact of densely local connection," Chinese Physics Letters, vol. 33, no. 2, pp. 28901-28904, 2016.

[31] M. Kitsak, S. Havlin, G. Paul, M. Riccaboni, F. Pammolli, and H. E. Stanley, "Betweenness centrality of fractal and nonfractal scale-free model networks and tests on real networks," Physical Review E, vol. 75, no. 5, p. 56115, 2007.

[32] C. Gao, X. Lan, X. Zhang, and Y. Deng, "A bio-inspired methodology of identifying influential nodes in complex networks," PLoS One, vol. 8, no. 6, Article ID e66732, 2013.

[33] J. S. Kim, K.-I. Goh, B. Kahng, and D. Kim, "Fractality and self-similarity in scale-free networks," New Journal of Physics, vol. 9, no. 6, p. 177, 2007.

[34] C. Dangalchev, "Residual closeness in networks," Physica A: Statistical Mechanics and Its Applications, vol. 365, no. 2, pp. 556-564, 2006.

[35] J. G. Restrepo, E. Ott, and B. R. Hunt, "Characterizing the dynamical importance of network nodes and links," Physical Review Letters, vol. 97, p. 94102, 2006.

[36] L. Y. Lü, C. H. Jin, and T. Zhou, "Similarity index based on local paths for link prediction of complex networks," Physical Review E, vol. 80, p. 46122, 2009. 
[37] P. L. Krapivsky and S. Redner, "Network growth by copying," Physical Review E, vol. 71, p. 36118, 2005.

[38] R. K. Nibbe, S. A. Chowdhury, M. Koyutürk, R. Ewing, and M. R. Chance, "Protein-protein interaction networks and subnetworks in the biology of disease," Wiley Interdisciplinary Reviews: Systems Biology and Medicine, vol. 3, no. 3, pp. 357-367, 2011.

[39] D. Wei, X. Zhang, and S. Mahadevan, "Measuring the vulnerability of community structure in complex networks," Reliability Engineering and System Safety, vol. 174, pp. 41-52, 2018.

[40] D. Bu, Y. Zhao, L. Cai et al., "Topological structure analysis of the protein-protein interaction network in budding yeast," Nucleic Acids Research, vol. 31, no. 9, pp. 2443-2450, 2003.

[41] D. Lusseau, K. Schneider, O. J. Boisseau, P. Haase, E. Slooten, and S. M. Dawson, "The bottlenose dolphin community of Doubtful Sound features a large proportion of long-lasting associations," Behavioral Ecology and Sociobiology, vol. 54, no. 4, pp. 396-405, 2003.

[42] J. J. Sun and S. K. Si, "Complex Network Algorithms and Applications," in National Defense Industry Press, X. Sun and S. Si, Eds., p. 22, Beijing, China, 2015.

[43] M. Gaertler and M. Patrignani, "Dynamic analysis of the autonomous system graph," in Proceedings of the IPS 2004, International Workshop on Inter-domain Performance and Simulation, pp. 13-24, Budapest, Hungary, March 2004.

[44] D. J. Watts and S. H. Strogatz, "Collective dynamics of 'smallworld' networks," Nature, vol. 393, no. 6684, pp. 440-442, 1998.

[45] R. Albert and A. L. Barabasi, "Statistical mechanics of complex networks," Review of Modern Physics, vol. 74, pp. 159-167, 2001.

[46] T. Carletti and S. Righi, "Weighted fractal networks," Physica A: Statistical Mechanics and Its Applications, vol. 389, no. 10, pp. 2134-2142, 2010.

[47] M. Kitsak, L. K. Gallos, S. Havlin et al., "Identification of influential spreaders in complex networks," Nature Physics, vol. 6, no. 11, pp. 888-893, 2010.

[48] A. Zeng and C.-J. Zhang, "Ranking spreaders by decomposing complex networks," Physics Letters A, vol. 377, no. 14, pp. 1031-1035, 2013.

[49] J.-G. Liu, Z.-M. Ren, and Q. Guo, "Ranking the spreading influence in complex networks," Physica A: Statistical Mechanics and Its Applications, vol. 392, no. 18, pp. 4154-4159, 2013.

[50] N. Blagus, L. Šubelj, and M. Bajec, "Self-similar scaling of density in complex real-world networks," Physica A: Statistical Mechanics and Its Applications, vol. 391, no. 8, pp. 2794-2802, 2012.

[51] D. J. Wei, Q. Liu, H.-X. Zhang, Y. Hu, Y. Deng, and S. Mahadevan, "Box-covering algorithm for fractal dimension of weighted networks," Scientific Reports, vol. 3, no. 1, p. 3049, 2013.

[52] L. Guo and X. Cai, "The fractal dimensions of complex networks," Chinese Physics Letters, vol. 26, p. 88901, 2009.

[53] I. Xenarios and D. Eisenberg, "Protein interaction databases," Current Opinion in Biotechnology, vol. 12, no. 4, pp. 334-339, 2001. 


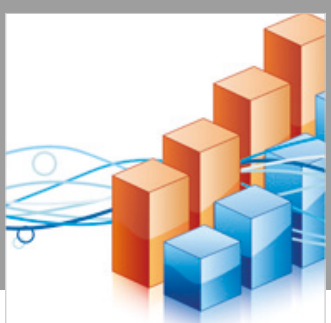

Advances in

Operations Research

\section{-n-m}
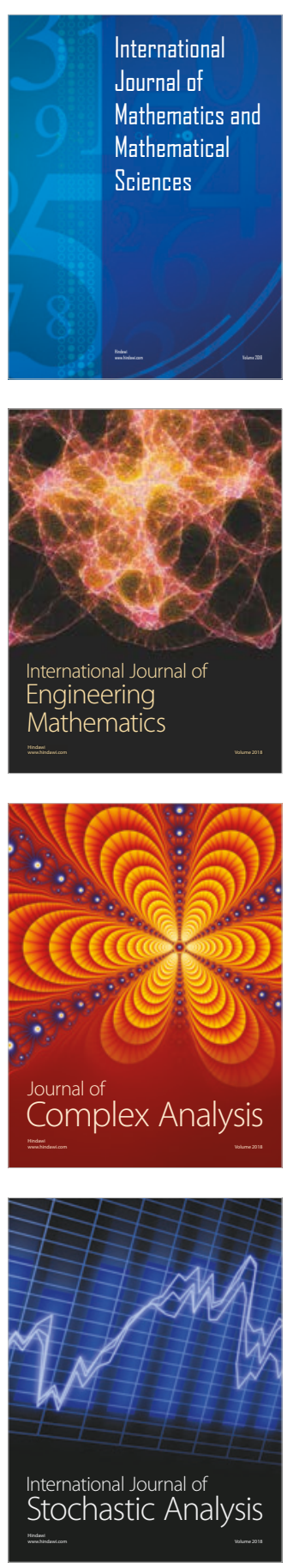
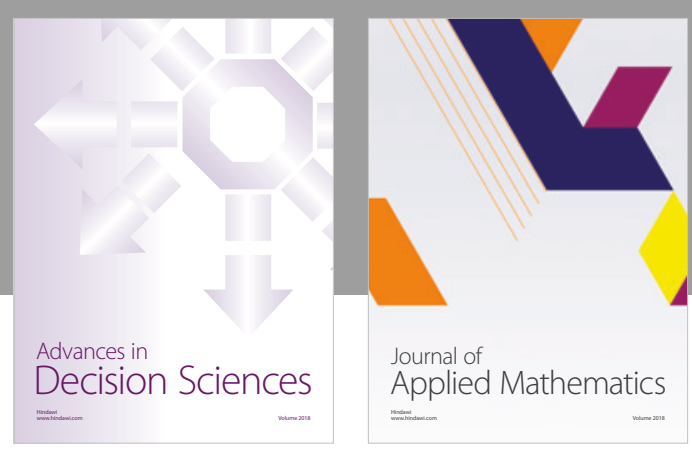

Journal of

Applied Mathematics
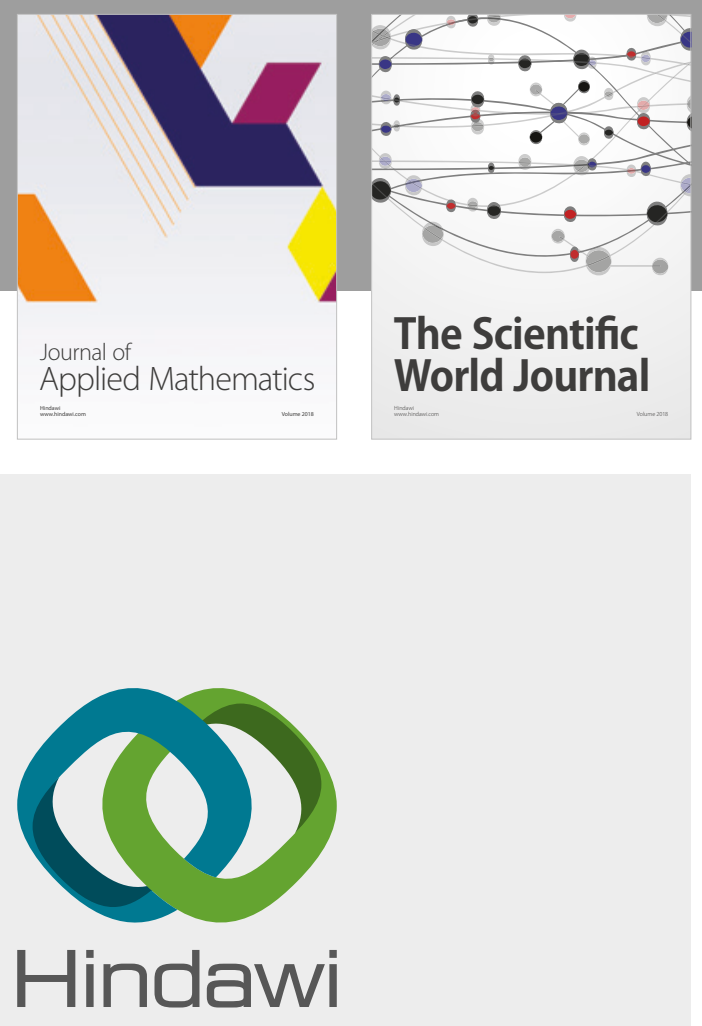

Submit your manuscripts at

www.hindawi.com

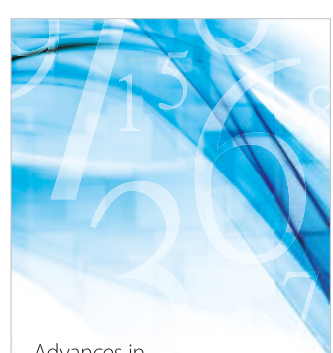

Advances in
Numerical Analysis
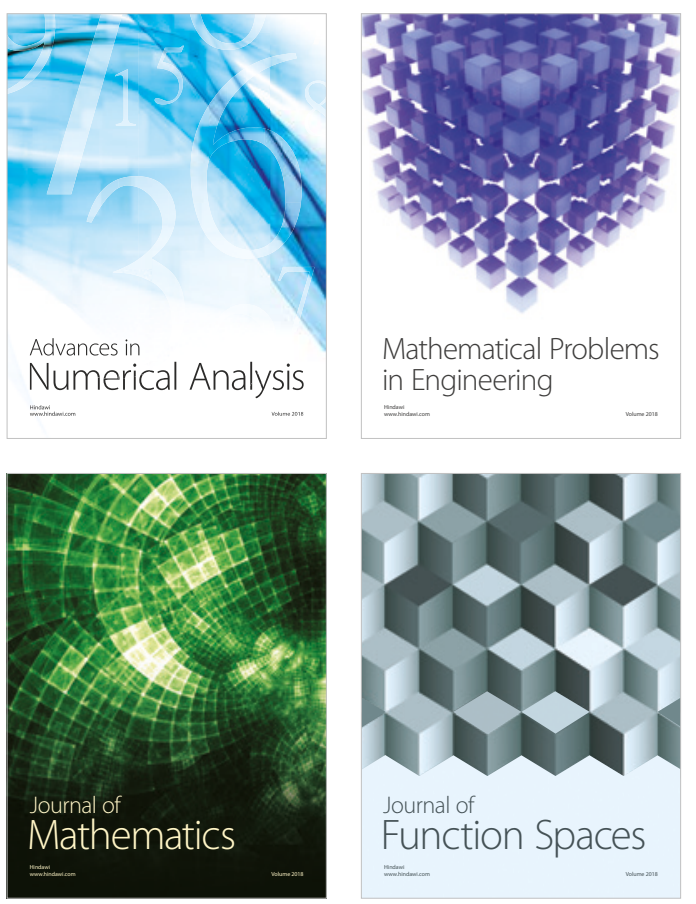

Mathematical Problems in Engineering

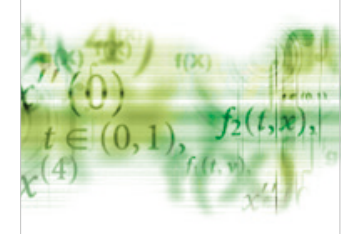

International Journal of

Differential Equations

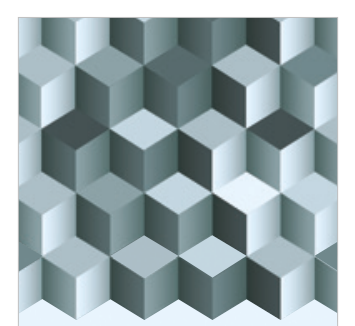

Journal of

Function Spaces
The Scientific

World Journal

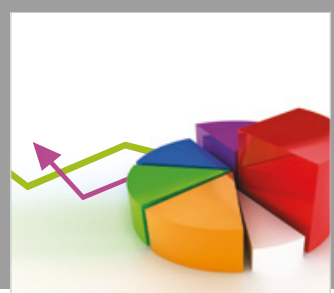

Journal of

Probability and Statistics
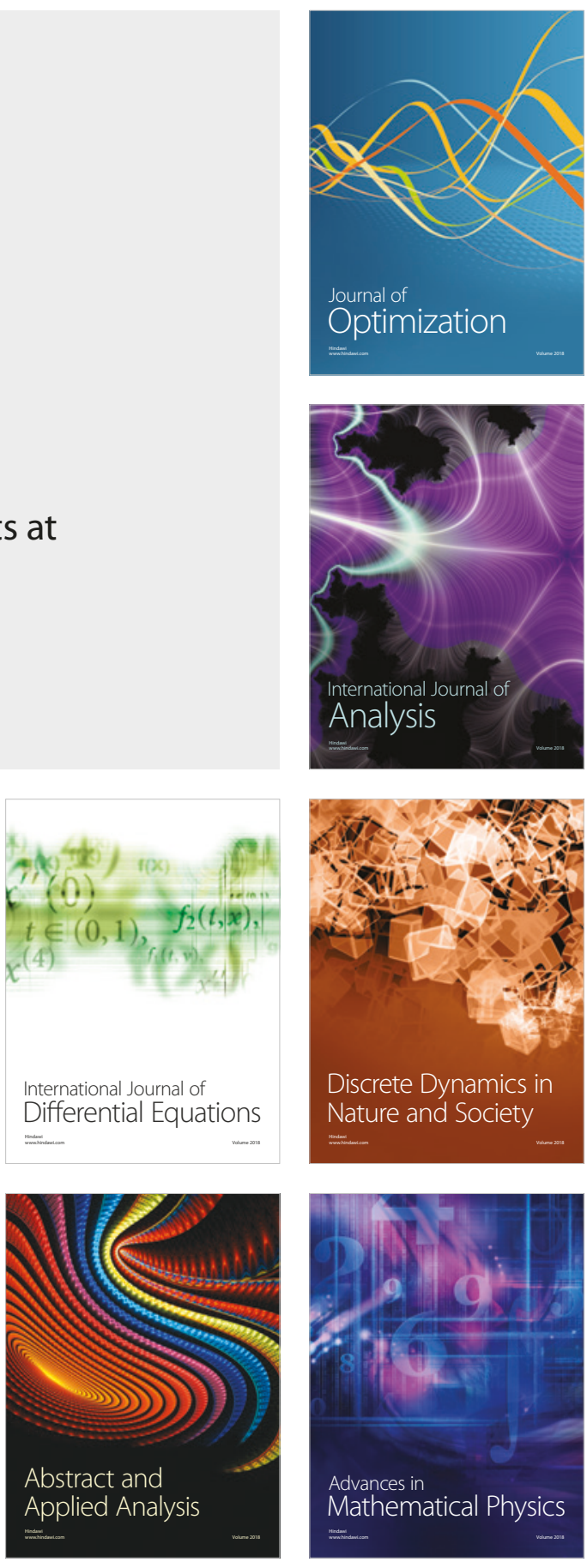